The Astrophysical Journal, 650:414-431, 2006 October 10

(C) 2006. The American Astronomical Society. All rights reserved. Printed in U.S.A.

\title{
DISCOVERY OF AN 86 AU RADIUS DEBRIS RING AROUND HD 181327
}

\author{
Glenn Schneider, ${ }^{1}$ Murray D. Silverstone, ${ }^{1}$ Dean C. Hines, ${ }^{2}$ Jean-Charles Augereau, ${ }^{3}$ Christophe Pinte, ${ }^{3}$ \\ François Ménard, ${ }^{3}$ John Krist, ${ }^{4}$ Mark Clampin, ${ }^{5}$ Carol Grady, ${ }^{6}$ David Golimowski, ${ }^{7}$ David Ardila, ${ }^{8}$ \\ Thomas Henning, ${ }^{9}$ Sebastian Wolf, ${ }^{9}$ and Jens Rodmann 9 \\ Received 2005 December 31; accepted 2006 June 6
}

\begin{abstract}
HST NICMOS PSF-subtracted coronagraphic observations of HD 181327 have revealed the presence of a ringlike disk of circumstellar debris seen in $1.1 \mu \mathrm{m}$ light scattered by the disk grains, surrounded by a diffuse outer region of lower surface brightness. The annular disk appears to be inclined by $31^{\circ} 7 \pm 1.6$ from face-on, with the disk major-axis P.A. at $107^{\circ} \pm 2^{\circ}$. The total $1.1 \mu \mathrm{m}$ flux density of the light scattered by the disk (at $1^{\prime \prime} .2<r<5$. 0 ) of $9.6 \pm 0.8 \mathrm{mJy}$ is $0.17 \% \pm 0.015 \%$ of the starlight. Seventy percent of the light from the scattering grains appears to be confined in a 36 AU wide annulus centered on the peak of the radial surface brightness (SB) profile $86.3 \pm 3.9$ AU from the star, well beyond the characteristic radius of thermal emission estimated from IRAS and Spitzer flux densities, assuming blackbody grains $(\approx 22 \mathrm{AU})$. The $1.1 \mu \mathrm{m}$ light scattered by the ring $(1)$ appears bilaterally symmetric, $(2)$ exhibits directionally preferential scattering well represented by a Henyey-Greenstein scattering phase function with $g_{\mathrm{HG}}=0.30 \pm 0.03$, and (3) has a median SB (over all azimuth angles) at the $86.3 \mathrm{AU}$ radius of peak SB of $1.00 \pm 0.07 \mathrm{mJy} \operatorname{arcsec}^{-2}$. No photocentric offset is seen in the ring relative to the position of the central star. A low SB diffuse halo is seen in the NICMOS image to a distance of $\sim 4^{\prime \prime}$. Deeper $0.6 \mu \mathrm{m}$ Hubble Space Telescope (HST) ACS PSF-subtracted coronagraphic observations reveal a faint $\left(V \approx 21.5 \mathrm{mag} \operatorname{arcsec}^{-2}\right)$ outer nebulosity at $4^{\prime \prime}<r<9^{\prime \prime}$, asymmetrically brighter to the north of the star. We discuss models of the disk and properties of its grains, from which we infer a maximum vertical scale height of 4-8 AU at the 87.6 AU radius of maximum surface density, and a total maximum dust mass of collisionally replenished grains with minimum grain sizes of $\approx 1 \mu \mathrm{m}$ of $\approx 4 M_{\text {Moon }}$.
\end{abstract}

Subject headings: circumstellar matter — infrared: stars — planetary systems: protoplanetary disks — stars: individual (HD 181327)

\section{INTRODUCTION}

Fifteen years elapsed between the acquisition of the prototypical scattered-light image of a debris disk around a young mainsequence star ( $\beta$ Pictoris; Smith \& Terrile 1984), offering strong support to the conjecture of the "Vega phenomenon" (far-IR emission in excess of a stellar photosphere from orbiting thermally emitting dust; Backman \& Paresce 1993), as suggested from earlier IRAS observations (Aumann et al. 1984), and the second example of a dusty circumstellar disk — in the form of a ring — seen by starlight illuminating its constituent grains (HR 4796A; Schneider et al. 1999). Advances in space- and ground-based high-contrast imaging capabilities have given rise to increasing, although still very small, numbers of scattered-light images of circumstellar debris systems. The very recent discoveries of a high surface brightness (SB) $\beta$ Pictoris-like nearly edge-on disk around the A star HD 32297 (Schneider et al. 2005, hereafter SSH05), and the very low SB 140 AU ring around an A star much older than $\beta$ Pictoris, i.e., Fomalhaut (Kalas et al. 2005), speak to the diversity in disk morphologies and scattering properties. While ringlike structures

\footnotetext{
1 Steward Observatory, University of Arizona, 933 North Cherry Avenue, Tucson, AZ 85721.

2 Space Science Institute, 4750 Walnut Street, Suite 205, Boulder, CO 80301.

3 Laboratoire d'Astrophysique de Grenoble, B.P. 53, 38041 Grenoble Cedex 9, France.

4 Jet Propulsion Laboratory, 4800 Oak Grove Drive, Mail Stop 183-900, Pasadena, CA 91109.

5 NASA Goddard Space Flight Center, Code 680, Greenbelt, MD 20771.

6 Eureka Scientific, 10813 Graeloch Road, Laurel, MD 20723-1124.

7 Johns Hopkins University, 3400 North Charles Street, Baltimore, MD 20218.

8 Spitzer Science Center, Pasadena, CA 91125.

9 Max-Planck-Institut fur Astronomie, Konigstuhl 17, D-69117 Heidelberg, Germany.
}

are obscured in scattered-light images of nearly edge-on disks, their intrinsic morphologies may be inferred by inversion of their radial SB profiles (e.g., Buitrago \& Mediavilla [1986], Pantin $\&$ Lagage [1996], and Augereau et al. [2001] for $\beta$ Pictoris and models presented by Krist et al. [2005] and Metchev et al. [2005] for AU Microscopii). We now report the discovery of a ringlike disk of circumstellar debris seen about the star HD 181327. This new addition to the growing menagerie of circumstellar debris disks is, in many respects, similar to the HR 4796A star-disk system. The recent imagery of a broader ring about the older solar analog, HD 107146 (Ardila et al. 2004), suggests that such structures are a common (if not prevalent) outcome of the planet formation process.

HD 181327 (HIP 95270) is an F5/F6 V star ( $T_{\text {eff }} \approx 6450 \mathrm{~K}$; Nordstrom et al. 2004; $\log g=4.5$ ) at $50.6 \pm 2.1 \mathrm{pc}$ (Perryman et al. 1997) with a Vega-like far-IR excess noted by Backman \& Paresce (1993). HD 181327 was identified as a main-sequence debris disk candidate by Mannings \& Barlow (1998) with $L_{\mathrm{IR}} / L_{*}=$ $0.2 \%$. Kalas et al. (2002) favored the Vega phenomenon interpretation of its far-IR excess based on its youth and its gas-depleted environment. The star's youth was first suggested from its potential membership in the $\sim 40$ Myr Tucanae association (Zuckerman $\&$ Webb 2000), but the star was later identified as a member of the younger ( $\sim 12 \mathrm{Myr}) \beta$ Pictoris moving group (Zuckerman \& Song 2004; Mamajek et al. 2004).

The conjecture of a stellar age of $\sim 10^{7} \mathrm{yr}$ is further supported by two independent observations. First, HD 181327 appears to exhibit common proper motion with the $7^{\prime}(0.1 \mathrm{pc}$ at $50 \mathrm{pc})$ distant HR 7329, also identified as a member of the $\beta$ Pictoris moving group by Zuckerman et al. (2001). Both stars have statistically identical Hipparcos parallaxes and (long baseline) Tycho 2 proper motions. HR 7329 is a zero-age main-sequence (ZAMS) A star 
TABLE 1

NiCMOS Observations of HD 181327 and PSF Reference Stars

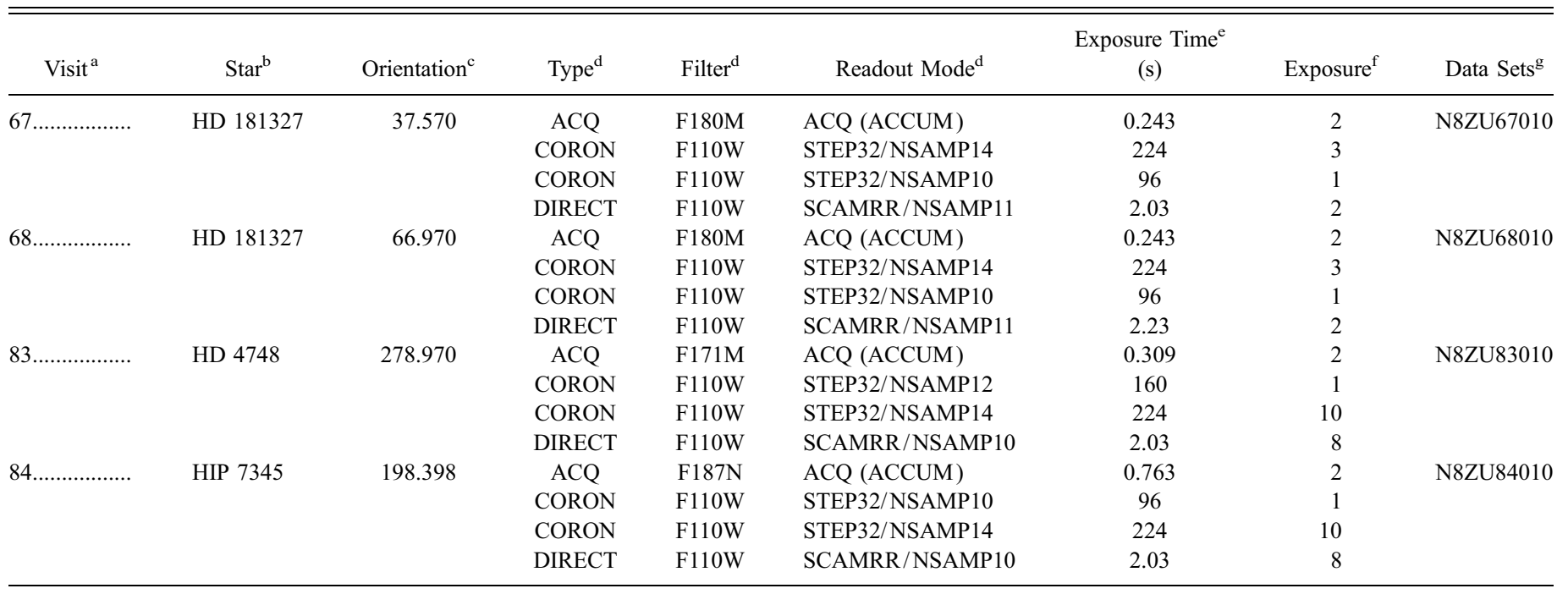

${ }^{\text {a }}$ HST GO 10177 Visit ID number.

b 2MASS magnitudes from Cutri et al. (2003). Spectral types from Perryman et al. (1997)-HD 181327: VT=7.095 $\pm 0.007, J=6.20 \pm 0.02, H=5.98 \pm 0.04$, $K=5.91 \pm 0.04$, F5/F6 V; HR 4748: $J=5.52 \pm 0.02, H=5.58 \pm 0.03, K=5.54 \pm 0.02$, B8 V; HIP 7345: $J=5.49 \pm 0.02, H=5.53 \pm 0.02, K=5.46 \pm 0.02$, A1 V.

c Position angle (degrees) of image $Y$-axis (east of north).

${ }^{d}$ See Noll et al. (2004) for NICMOS image/readout modes and filters.

exposure time for each exposure (before combining).

${ }^{\mathrm{f}}$ Number of exposures.

${ }^{g} H S T$ archive data set ID.

possessing a close substellar companion ${ }^{10}$ with an isochronal age of 10-30 Myr (Lowrance et al. 2000). Second, HD 181327's Hipparchos-derived $B-V$ color index of +0.480 is completely consistent with an unreddeded F5-F6 star. ${ }^{11}$ This then is in agreement with HD 181327's $e(b-y)=0.002 \mathrm{mag}$ (Nordstrom et al. 2004), and the stellar luminosity is then $3.1 \pm 0.3 L_{\odot}$, from which the stellar age estimated using evolutionary tracks by D'Antona \& Mazzitelli (1997), Palla \& Stahler (2001), and Baraffe et al. (1998) is consistent with $\sim 20$ Myr.

Assuming blackbody grains, Zuckerman \& Song (2004) suggested a thermal equilibrium temperature of $65 \mathrm{~K}$ for the IRemitting dust at a characteristic orbital radius of $35 \mathrm{AU}$ from HD 181327. Neuhäuser et al. (2003), using $N N T+$ SHARP-I and Sof I, and Chauvin et al. (2003), with ESO 3.6 m AO+ADONIS/ SHARPII, conducted searches for a companion candidate to HD 181327, but found none.

\section{NICMOS OBSERVATIONS AND DATA PROCESSING}

During HST Cycle 13 we conducted a NICMOS coronagraphic imaging survey (HST GO 10177; PI: Schneider) of 26 main-sequence stars, $\sim 10 \mathrm{Myr}$ and older, with strong far-IR excess emission, including HD 181327, to identify and provide spatially resolved scattered-light images of previously suspected, but unimaged, dusty circumstellar disks (SSH05). HD 181327 was observed at two field orientations in a single spacecraft orbit on 2005 May 2 UT using $H S T$ NICMOS camera 2 (scale: $\sim 75.8$ mas

\footnotetext{
${ }^{10}$ The total mass of HD $181327+$ HR $7329 \mathrm{~A} / \mathrm{B}$ is $\sim 3.8 M_{\odot}$, and stellar binaries with separations $\sim 0.1 \mathrm{pc}$ are not unheard of with similar systemic masses. Hence, in the light of recent discussion on brown dwarf formation and binary populations (e.g., see Close et al. 2003, Fig. 15; Burgasser et al. 2006) one might conjecturally consider HD $181327+$ HR 7329 A/B to be a triple system.

${ }_{11}$ In the Hipparcos catalog, the F5 and F6 stars within $75 \mathrm{pc}$ with parallaxes of $\mathrm{S} / \mathrm{N} \geq 8$ have median $B-V$ color indices of +0.464 and +0.496 , respectively. Hence, there appears to be no evidence for reddening for HD 181327 (unsurprising, given its distance and environment).
}

pixel $\left.^{-1}\right)$ with F110W $\left(\lambda_{\text {eff }}=1.104 \mu \mathrm{m}, \mathrm{FWHM}=0.5915 \mu \mathrm{m}\right)$ filter direct and coronagraphic imaging and supporting F180M $\left(\lambda_{\text {eff }}=1.7968 \mu \mathrm{m}, \mathrm{FWHM}=0.0684 \mu \mathrm{m}\right)$ target acquisition exposures. Table 1 provides observational details for our program stars and for the point-spread function (PSF) reference stars that were used to construct the PSF-subtracted imagery discussed in $\S 2.1$. Details of the observing strategy, data reduction and calibration methodologies, and image combination processes, which we apply to all debris disk candidate (and reference PSF) star observations in our HST GO 10177 survey are discussed by SSH05, to which we refer the reader for details.

\subsection{NICMOS PSF Subtractions}

To further reduce the level of the residual instrumentally diffracted and scattered circumstellar light, beyond the improvement in image contrast afforded by the coronagraph itself, we subtract astrometrically registered, flux-scaled images of coronagraphic reference PSFs of nondisk stars of similar spectral energy distributions (SED) from each of the two (one at each field orientation) calibrated combined images of HD 181327. We follow the precepts of the two-orientation coronagraphic PSF subtraction process described by SSH05. We selected 10 candidate PSF stars with near-IR colors similar to HD 181327. Two of these were specifically programmed very bright PSF calibration stars with no IRAS-detected excess emission, namely, $\mathrm{HR} 4748(J=5.52 \mathrm{mag})$ and HIP $7345(J=5.49 \mathrm{mag})$, each observed at a single field orientation using a full spacecraft orbit and yielding a very high signal-to-noise ratio $(\mathrm{S} / \mathrm{N})$. Eight other PSF template candidates were selected from GO 10177 survey targets that exhibited no evidence of scattered-light excesses from circumstellar dust. Each of these eight stars was observed at two field orientations (thus providing two PSF templates each), but was much less deeply exposed than our PSF calibration targets, due to their lesser brightness and the detailed nature of the observing/integration 


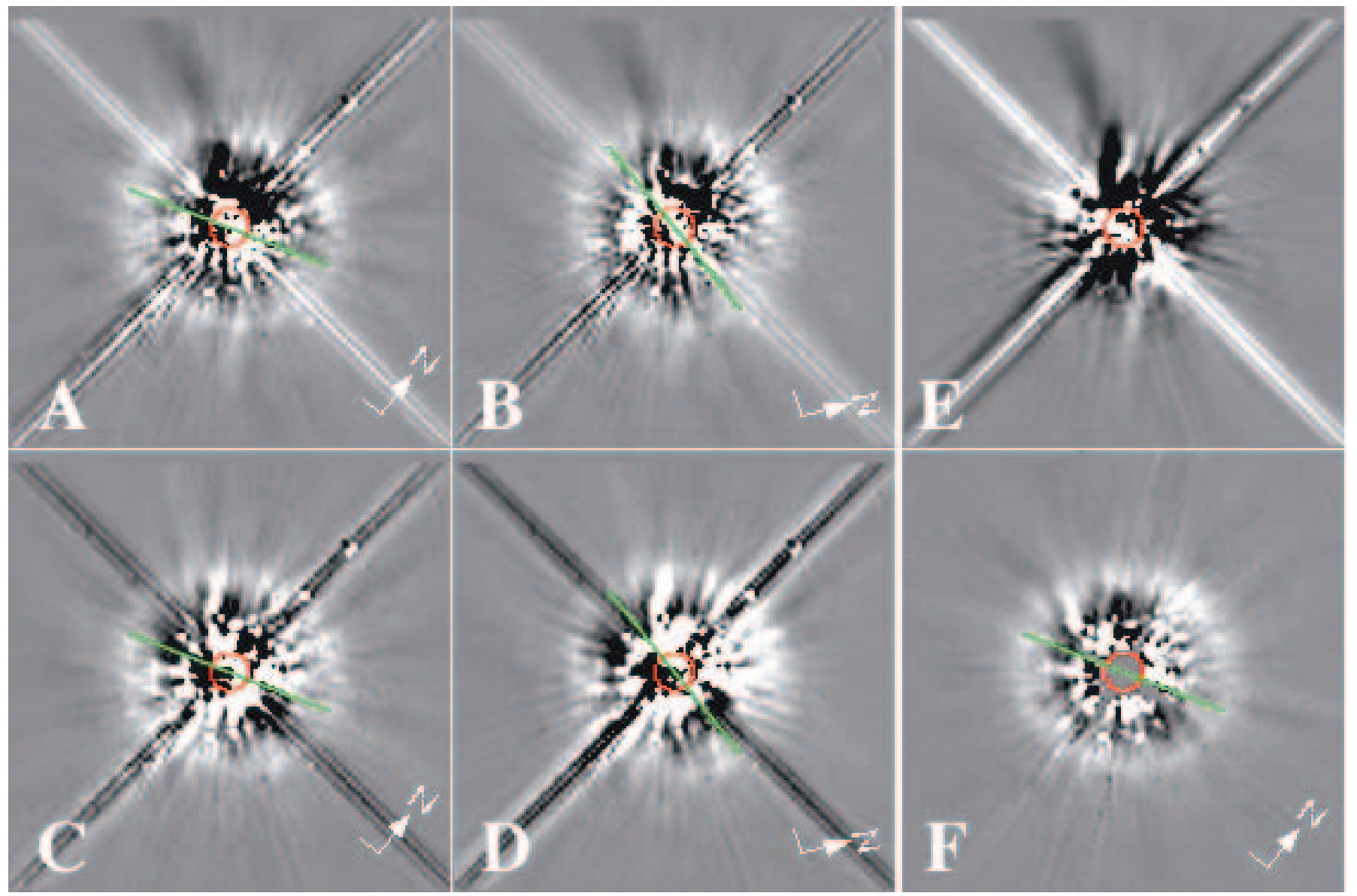

FIG. 1. - $(a-d)$ Representative astrometrically registered, flux-scaled difference images, using two different PSF template stars [HIP 7345 in $(a-b)$ and HIP 4748 in $(c-d)$ ], with HD 181327 observed at two field orientations differing by $29^{\circ} .9$ (absolute orientations with respect to north as indicated), illustrating the robustness of NICMOS PSF-subtracted coronagraphy in revealing the HD 181327 circumstellar debris ring. (e) Same-scaled and registered PSF minus PSF subtraction of the two template stars. All images are shown with a symmetric bipolar stretch of \pm 10 counts s$^{-1}$ pixel $^{-1}$ ( $\mathrm{F} 110 \mathrm{~W} \mathrm{SB}$ of $\pm 2.19 \mathrm{mJy} \operatorname{arcsec}^{-2}$ ) to show the nature (and intensity) of the PSF subtraction residuals. Differential diffractive and scattering artifacts remain spatially fixed on the detector with the spacecraft reorientation, but vary in intensity and phase primarily due to spacecraft "breathing" (Schneider et al. 2001). Note, for example, the diagonal diffraction spikes from the HST secondary mirror support and a commonly occurring diffuse coronagraphic scattering feature (known as "the smokestack," slightly curving to the upper left, dark in $[a],[b]$ and $[e]$ ), which appears in conjunction with a more intense "finger" closer to and above the coronagraphic hole (red circles), particularly bright in $(c)$ and $(d)$. $(f)$ Combination of images in $(a-d)$, medianed with equal weight after masking the diffraction spike artifacts in the individual images and rotating all to the same celestial orientation. All panels are $7^{\prime \prime} \times 7^{\prime \prime}$. Green lines indicate the disk major axis.

time strategy for these targets. All 36 HD 181327 minus PSF template subtractions revealed an elliptical ringlike disk that rotated in the celestial frame, following the $29^{\circ} .9$ difference in target orientation between the two HD 181327 observational visits (e.g., Figs. $1 a-1 d$ ). PSF minus PSF subtractions exhibited no such structure (e.g., Fig. 1e), while in all cases rotationally invariant features due to instrumental scattering and diffraction remained fixed in the frame of the detector.

The four images resulting from subtracting the HR 4748 and HIP 4375 high S/N reference PSFs from each of the two images of HD 181327 (differing in orientation $29^{\circ} .9$ ) were of higher photometric efficacy than those obtained using the serendipitous ensemble of PSFs derived from the GO 10177 survey targets and generally were more devoid of azimuthally anisotropic high- and midspatial frequency artifacts arising from aliasing in imperfect PSF subtractions. The HD 181327 debris disk image, which we discuss in this paper, was created using these four PSF-subtracted images. The four images were median-combined with equal weight after alignment, rotation to a common orientation, and masking regions in individual images affected by diffraction spikes from the HST secondary mirror support structure (see Fig. $1 f$ ). The final NICMOS image, shown in Figure $1 f$ (and Fig. $2 a$ ) and dis- cussed in $\S \S 5$ and 6 , has a total integration time of $1536 \mathrm{~s}$ in most pixels.

\section{ACS OBSERVATIONS}

HD 181327 was observed earlier in HST GTO program 9987 (PI: Ford) on 2003 November 2 UT using the HST Advanced Camera for Surveys High Resolution Camera (ACS HRC; scale $\sim 25$ mas pixel $^{-1}$ ) in direct and coronagraphic imaging modes. Two $5 \mathrm{~s}$ exposures were taken without the coronagraph in the F606W ("wide" $V$ band) filter. After a 0.5 s F502N acquisition exposure, the star was placed behind the 0 ". 9 radius coronagraphic occulting spot, and two 1235 s F606W exposures were taken. The ACS images were calibrated by the HST OPUS ${ }^{12}$ pipeline, and the two coronagraphic exposures were combined with cosmic-ray rejection.

\subsection{ACS Coronagraphy and PSF Subtraction}

By suppressing the diffraction pattern of the occulted star, the ACS coronagraph reduces the SB of the wings of the stellar PSF by about a factor of 7-10. As with NICMOS, an additional gain

12 See http://www.stsci.edu/resources/software_hardware/opus. 

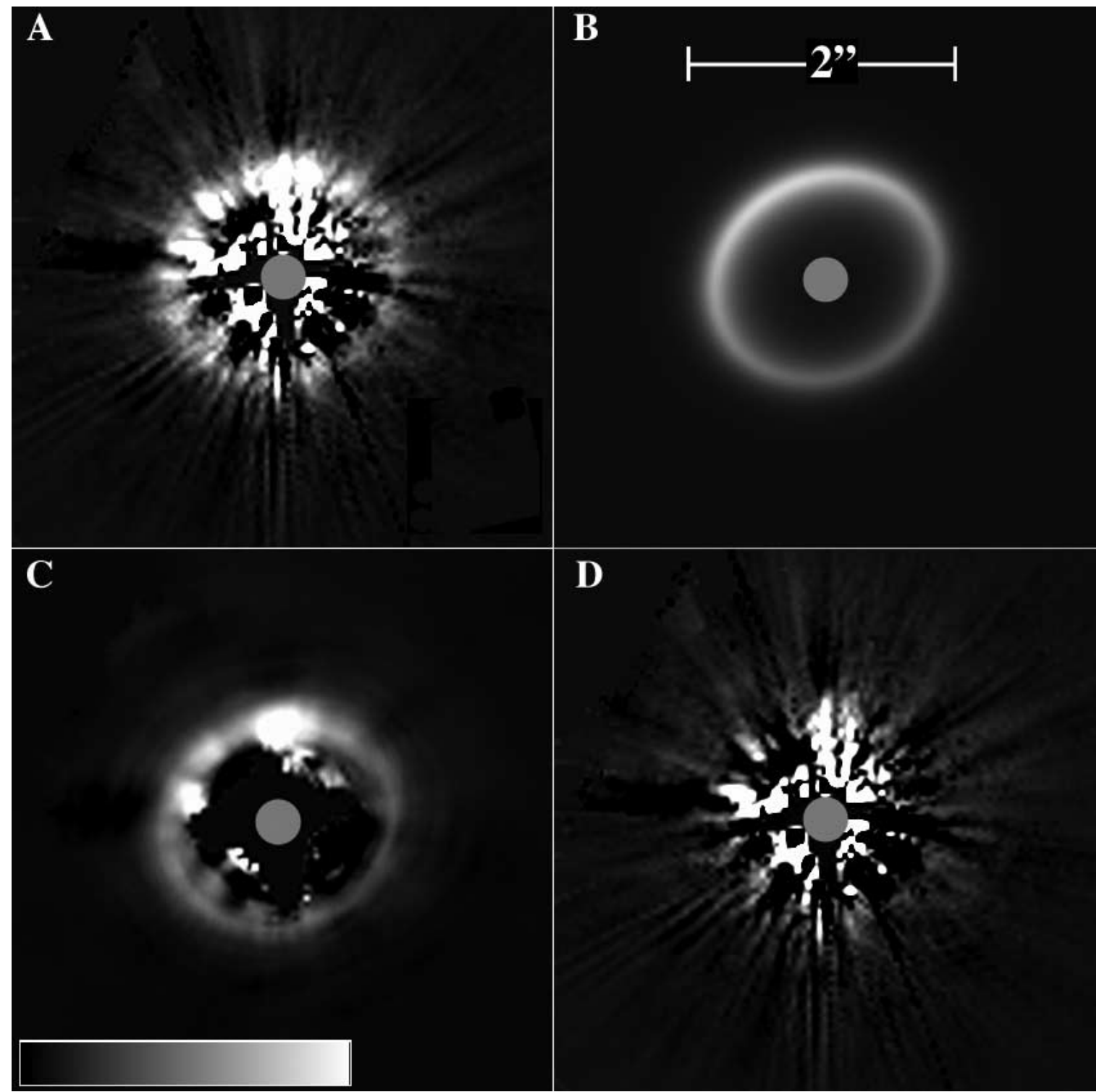

FIG. 2.- (a) NICMOS $1.1 \mu \mathrm{m}$ two-orientation combined PSF-subtracted coronagraphic image of the HD 181327 debris ring (same image data as Fig. $1 f$ ). (b) Bestfit photometric model, as discussed in $\S 5.4$ of the text. $(c)$ Observed image (from $[a]$ ) after low-pass filtering with a $9^{\circ}$ elliptical boxcar kernel. $(d)$ Residuals after subtracting $(b)$ from $(a)$. All panels are shown at the same linear display stretch, -0.5 (black) to +10 (white) counts s ${ }^{-1}$ pixel $^{-1}\left(-0.11\right.$ to $+2.19 \mathrm{mJy}$ arcsec $\left.{ }^{-2}\right)$, and scale (as indicated). Small ( $\left.r=0^{\prime \prime} \cdot 3\right)$ gray circles are centered on the location of the star.

in image contrast can be achieved by subtracting the underlying stellar PSF using a coronagraphic image of another occulted star of similar color, reducing the remaining "background" by factors of 10-100. Additional information on ACS coronagraphic performance is presented in $\S 6.2$ of Gonzaga et al. (2005).

In the case of HD 181327, observations of the F5 V star HD 191089 were used to create a template PSF image for subtraction. HD 191089 was imaged in a single spacecraft orbit immediately following the observations of HD 181327, and a very similar imaging and data-processing strategy was employed. Two $6 \mathrm{~s}$ direct and two 1005 s coronagraphic $(r=0$ ".9 occulting spot) exposures were obtained with the F606W filter. The HD 191089 PSF template was scaled to the flux density of HD $181327 .{ }^{13}$ After

13 To establish the flux density scaling, for both stars the number of saturated pixels in the PSF core was multiplied by $165,000 e^{-}$pixel $^{-1}$ (full-well depth of an HRC CCD pixel) and added to the flux density measured within a $1^{\prime \prime}$ radius aperture. Charge in excess of 1 pixel full-well is transferred, without significant inefficiency, to neighboring nonsaturated pixels. For additional information see $\S \S 4.2 .6$ and 4.3.1 of Gonzaga et al. (2005), Gilliland (2004), and Bohlin et al. (2003). finite-aperture photometric and geometric distortion corrections, the target-to-PSF scaling obtained agreed within $2 \%$ of the expected value based on the $V$-band magnitudes of the stars. The subtraction of HD 181327 was optimized by iteratively shifting and subtracting the flux-scaled HD 191089 image until residuals induced by misregistration, beyond $2^{\prime \prime}$ from the occulted star, were minimized and spatially symmetric. This process coregisters the stars to $\sim 0.05 \mathrm{HRC}$ pixels (1.25 mas). The final subtracted image (shown in Fig. 3 at three different image scales and display stretches) was corrected for the well-characterized geometric distortion in the HRC.

\subsection{ACS Image Data and Caveats}

Near the coronagraphic obscuration, a residual halo of starlight unsuppressed by the ACS coronagraph arises from instrumental scattering (seen as radial streaks) and diffraction by the occulter itself (seen as concentric rings centered on the occulter). The scattered-light streaks are relatively insensitive to star-toocculter positioning, but the ringlike diffracted light distribution 

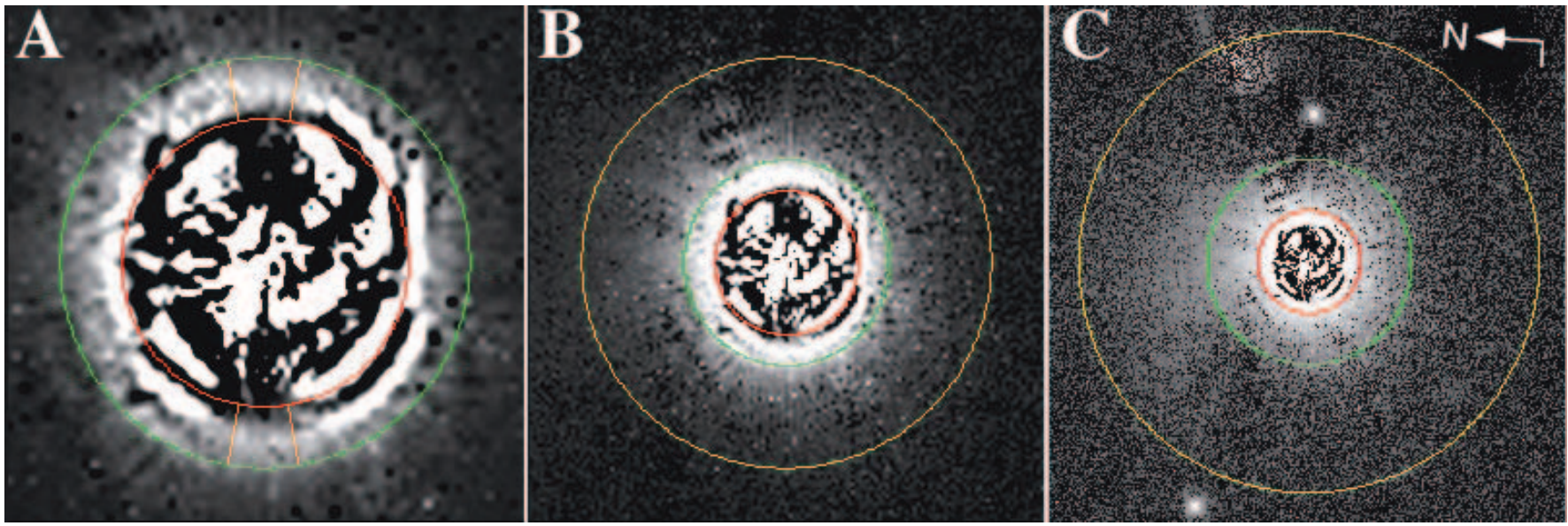

FIG. 3. - ACS $0.6 \mu \mathrm{m}$ PSF-subtracted coronagraphic image of HD 181327. (a) 2".5 52 ".5 field, $r=1$ 1"4 (red), $r=2$ ".0 ( green), -0.1 to $+1.5 e^{-} \mathrm{s}^{-1}$ pixel $^{-1}$ linear display. The disk is reliably seen only within $\pm 10^{\circ}$ of the image $+Y$-axis (P.A. $=274.9$; arcs bounded in orange). $(b) 5^{\prime \prime} \times 5^{\prime \prime}$ field, $r=1^{\prime \prime} 4($ red $), r=2^{\prime \prime}($ green $), r=4^{\prime \prime}$ (yellow), $0-1 e^{-} \mathrm{s}^{-1}$ pixel $^{-1}$ square root display. (c) $10^{\prime \prime} \times 10^{\prime \prime}$ field, $r=2^{\prime \prime}$ (red), $r=4^{\prime \prime}$ (green), $r=9^{\prime \prime}$ (yellow), $[-3.3]$ to $[+0.0] e^{-} \mathrm{s}^{-1}$ pixel ${ }^{-1}$ logarithmic display. Within $\pm 45^{\circ}$ of north and $4^{\prime \prime}-9^{\prime \prime}$ from the star a faint nebulosity can be seen, with some suggestion of curvature toward the east.

is sensitive to the location of the star within the footprint of the occulter. The ACS PSF-subtracted image of HD 181327 minus HD 191089 (after flux-scaling and image registration) shows instrumentally induced residuals beyond the edge of the occulting spot. The residuals are dominated by diffraction rings predominantly arising from differences in the positioning of the two stars behind the occulter. The registration of the HD 181327 and HD 191089 images required a larger than typical translation (30.1 mas) of the reference PSF, indicative of a target centration error in the acquisition process (assuming that the position of the occulting spot remained stable over the short period of the two-orbit sequential observations of both stars). The position error was much greater along the image horizontal (with respect to the detector), so the PSF subtraction residuals are also larger along that direction. Due to the asymmetry of the PSF subtraction residuals near the edge of the spot, (1) the HD 181327 disk can be detected reliably from 1 ." $4<r<2$ ".0 from the star only within $\sim \pm 10^{\circ}$ arcs centered on the image $Y$-axis on opposite sides of the star (Fig. $3 a$ ) and (2) from $2^{\prime \prime}<r<4^{\prime \prime}$ a diffuse halo is seen (Fig. 3b), although its nearly circular symmetry and ringlike substructures might implicate a residual contribution of instrumental origin (from imperfect PSF subtraction) that is somewhat difficult to quantify.

Further from the coronagraphic occulting spot, from $4^{\prime \prime}<$ $r<9^{\prime \prime}$ (well beyond the distance leading to PSF subtraction artifacts discussed in items 1 and 2 above), within $\pm 45^{\circ}$ of north, a faint nebulosity with a mean SB of $V \approx 21.5 \mathrm{mag} \operatorname{arcsec}^{-2}$ is seen (Fig. 3c).

\section{SPITZER MIPS AND IRAS PHOTOMETRY}

New 24, 70, and $160 \mu \mathrm{m}$ imaging photometric observations of HD 181327 were obtained with the MIPS instrument on the Spitzer Space Telescope on 2004 April 6 in GTO program 72 (PI: F. Low). We use the photometric measures derived from these observations, along with IRAS Faint Source Catalog (FSC) 25,60 , and $100 \mu \mathrm{m}$ photometry, to better define the thermal SED of the HD 181327 disk (see Fig. 4; Table 2). With these data we find a slightly larger thermal IR excess, $L_{\mathrm{IR}} / L_{*}=0.25 \%$, than Mannings \& Barlow (1998) and a somewhat warmer blackbody equilibrium temperature for the disk grains than the $65 \mathrm{~K}$ suggested by Zuckerman \& Song (2004). A least-squares blackbody fit to the $24 \mu \mathrm{m}$ and longer wavelength flux densities indicates an equilibrium temperature of $77 \pm 5 \mathrm{~K}$ for the thermally emitting grains (after subtracting the small underlying contribution from the stellar photosphere). Following Zuckerman \& Song (2004), the warmer grain temperature would also suggest - for grains emitting as blackbodies - a smaller characteristic orbital radius of $\approx 22 \mathrm{AU}$. The $12 \mu \mathrm{m}$ emission detected by IRAS is not in excess of the stellar photospheric level (see Fig. 4), as confirmed from a recent Spitzer IRS spectrum of HD 181327 (Chen et al. 2006).

\section{THE HD 181327 RINGLIKE DISK}

The NICMOS 1.1 $\mu \mathrm{m}$ PSF-subtracted image of the HD 181327 disk probes closer to the star than the ACS $0.6 \mu \mathrm{m}$ imagery at all azimuth angles $\left(\theta\right.$, with $\theta=0^{\circ}$ at the position angle closest to the line of sight toward the Earth; see $\S 5.1$ ). Because the effective inner radius of the ACS image was beyond the peak of the radial SB distribution at most azimuth angles (as revealed by the

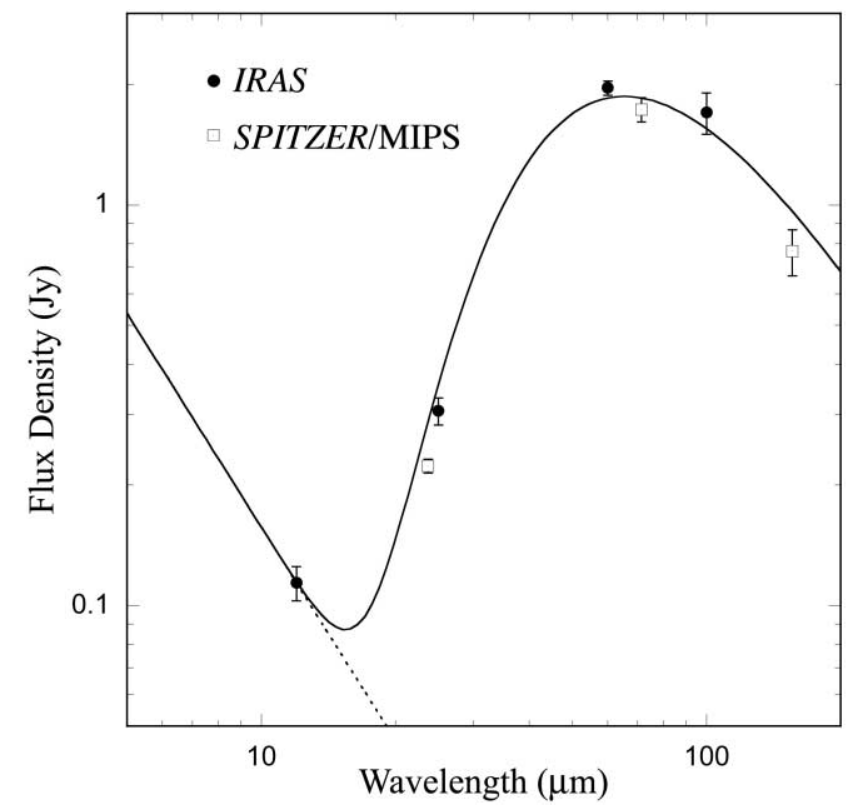

FIG. 4. - HD 181327 spectral energy distribution (SED) from color-corrected Spitzer MIPS and IRAS photometry (see Table 2). A least-squares blackbody fit to the photometric data indicates a grain equilibrium temperature of $77 \pm 5 \mathrm{~K}$. The black line is the sum of the $77 \mathrm{~K}$ fit and a blackbody of $T_{\text {eff }}=6450 \mathrm{~K}$ for the stellar photosphere (dotted line). 
TABLE 2

Spitzer MIPS and IRAS Thermal IR Flux Denisties

\begin{tabular}{clccc}
\hline \hline $\begin{array}{c}\text { Wavelength } \\
(\mu \mathrm{m})\end{array}$ & Source & $\begin{array}{c}\text { Flux Density }^{\mathrm{b}} \\
(\mathrm{Jy})\end{array}$ & $\begin{array}{c}\text { Uncertainty } \\
(\%)\end{array}$ & $\begin{array}{c}\text { Color-corrected } \\
(\mathrm{Jy})\end{array}$ \\
\hline $12 \ldots \ldots \ldots \ldots \ldots$. & IRAS & 0.164 & 10 & $0.114 \pm 0.011$ \\
$23.68 \ldots \ldots \ldots \ldots$. & MIPS & 0.220 & 4 & $0.223 \pm 0.009$ \\
$25 \ldots \ldots \ldots \ldots \ldots \ldots$. & IRAS & 0.248 & 8 & $0.306 \pm 0.024$ \\
$60 \ldots \ldots \ldots \ldots \ldots$. & IRAS & 1.86 & 4 & $1.96 \pm 0.08$ \\
$71.42 \ldots \ldots \ldots \ldots$. & MIPS & 1.58 & 7 & $1.73 \pm 0.12$ \\
$100 \ldots \ldots \ldots \ldots \ldots$. & IRAS & 1.72 & 12 & $1.70 \pm 0.20$ \\
$155.9 \ldots \ldots \ldots \ldots$. & MIPS & 0.75 & 12 & $0.77 \pm 0.09$ \\
\hline
\end{tabular}

${ }^{\text {a }}$ Weighted average wavelengths for MIPS; see http://ssc.spitzer.caltech .edu/mips/dh.

b IRAS flux densities from the FSC.

c IRAS $12 \mu \mathrm{m}$ flux density for $6450 \mathrm{~K}$ stellar photosphere. Flux densities for all other bands presume $70 \mathrm{~K}$ blackbody circumstellar grains. See http://irsa .ipac.caltech.edu/IRASdocs/exp.sup/ch6/tabsupC6.html and http://ssc.spitzer .caltech.edu $/ \mathrm{mips} / \mathrm{dh} / \mathrm{mipsdatahandbook3.2.pdf.}$

NICMOS image), we determined the systemic geometric and overall photometric properties of the HD 181327 disk from the NICMOS image alone.

The HD 181327 debris disk seen in the NICMOS $1.1 \mu \mathrm{m}$ image is in the form of an inclined ring (Fig. 2a). The radial SB of the ring declines monotonically from the (deprojected) radius of its peak brightness, $R_{\text {peak }}$, both inward (appearing cleared toward the center) and outward (appearing bounded or truncated) from $R_{\text {peak }}$.

To establish the geometry of the distribution of debris-scattered light in the HD 181327 ring, we fit image isophotes to nested ellipses and simultaneously solved for the ring axial lengths, majoraxis orientation, and photocenter by iterative $\chi^{2}$ minimization to the NICMOS image. The location of the star (occulted by the coronagraph) was determined using acquisition offset slew vectors downlinked in the engineering telemetry applied to the star's position measured from the well-exposed and critically sampled, preslew target acquisition images with a precision of $\lesssim 1 / 20$ pixel ( 4 mas; $0.2 \mathrm{AU}$ at $50 \mathrm{pc}$ ).

\subsection{Geometry of the Ring}

Ring geometry. - Assuming intrinsic circular symmetry, we found the ring inclination, $i=31^{\circ} .7 \pm 1^{\circ} .6$, from a face-on view- ing geometry, with the disk major axis oriented at a P.A. of $107^{\circ} \pm 2^{\circ}$ east of north. The best elliptical fits find the radial peak in SB at a semimajor-axis length of $22.5 \pm 0.3$ pixels $(1$.706 \pm 0 ".023). Given HD 181327's annual parallax of $19.77 \pm 0.81$ mas, we find the radius of the debris ring, $R_{\text {peak }}=86.3 \pm 3.7 \mathrm{AU}$.

Bilateral symmetry.-Figure 5 shows the disk deprojected from the plane of the sky with the major axis on the image horizontal and the location of the occulted star marked. The HD 181327 ring SB appears to be bilaterally symmetric, and the disk SB peaks radially at the same distance at all azimuths. The absence of any significant departure from circular symmetry is illustrated in a lowpass filtered image (Fig. 5b) and the corresponding isophotal map of the deprojected disk (Fig. $5 c$ ). The ring appears to be centered on the star to within a measurement uncertainty of $\sim 0.25$ pixels ( $1 \mathrm{AU}$ at $50 \mathrm{pc}$ ).

\subsection{Photometry of the Disk}

The $1.1 \mu \mathrm{m}$ flux density. - Because of residual "noise" in the PSF subtraction close to the star, there is little useful information at $r<1$."2 from the star. The flux density from the ring dominates the subtraction residuals at larger radii. We measured the total $1.1 \mu \mathrm{m}$ flux density of the disk at $1.2<r<5$ ". 0 as $9.6 \pm 0.8 \mathrm{mJy}$. The main contributor to the $\sim 8 \%$ measurement error arises from imperfect removal of the underlying stellar PSF by under- or oversubtraction due to uncertainties in flux scaling. We assess the magnitude of this error from null PSF-PSF subtractions of the reference stars, scaled to the flux density of HD 181327 (e.g., see Schneider et al. 2001; SSH05). There may be more dust-scattered light interior to this radius. However, as shown below, the radial SB of the disk declines steeply inward from the brightest part of the ring at 1 .'71 (deprojected). The small area interior to $1^{\prime \prime} .2$ is likely to contribute only weakly to the total light-scattered disk flux density.

The $1.1 \mu \mathrm{m}$ scattering fraction.-Our direct (noncoronagraphic) images of HD 181327 were saturated in their PSF cores (as expected). Hence, we transform the 2MASS $J=6.20$ magnitude of HD 181327 to the NICMOS F110W band using the CALCPHOT task in the STSDAS/SYNPHOT synthetic photometry package. ${ }^{14}$ Using an F6 V input spectrum, we find that the

\footnotetext{
14 See http://www.stsci.edu/resources/software_hardware/stsdas/synphot.
}
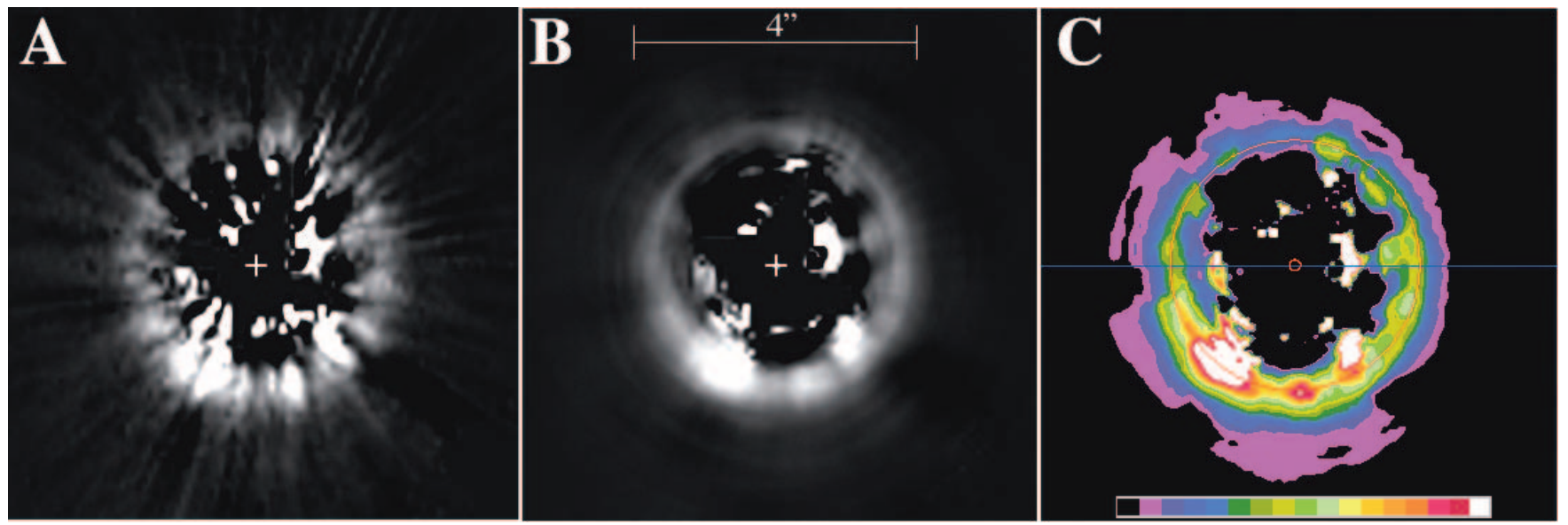

FIG. 5. - HD 181327 debris ring projected to a face-on geometry, while conserving the total flux density. In this figure ( $a-b)$ correspond to (a) and (c) in Fig. 2, but

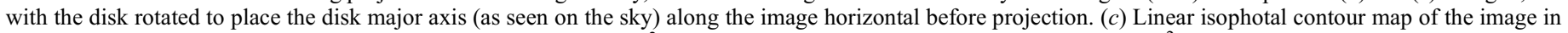

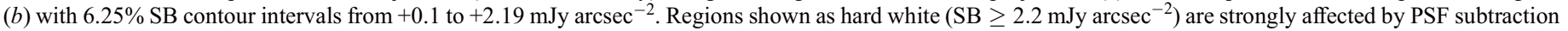

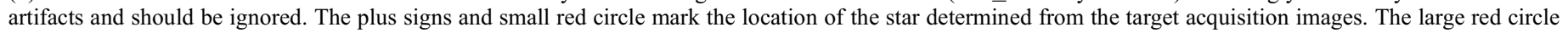
indicates the distance at which the SB peaks radially, and the blue line indicates the major axis of the ring projected onto the sky. 


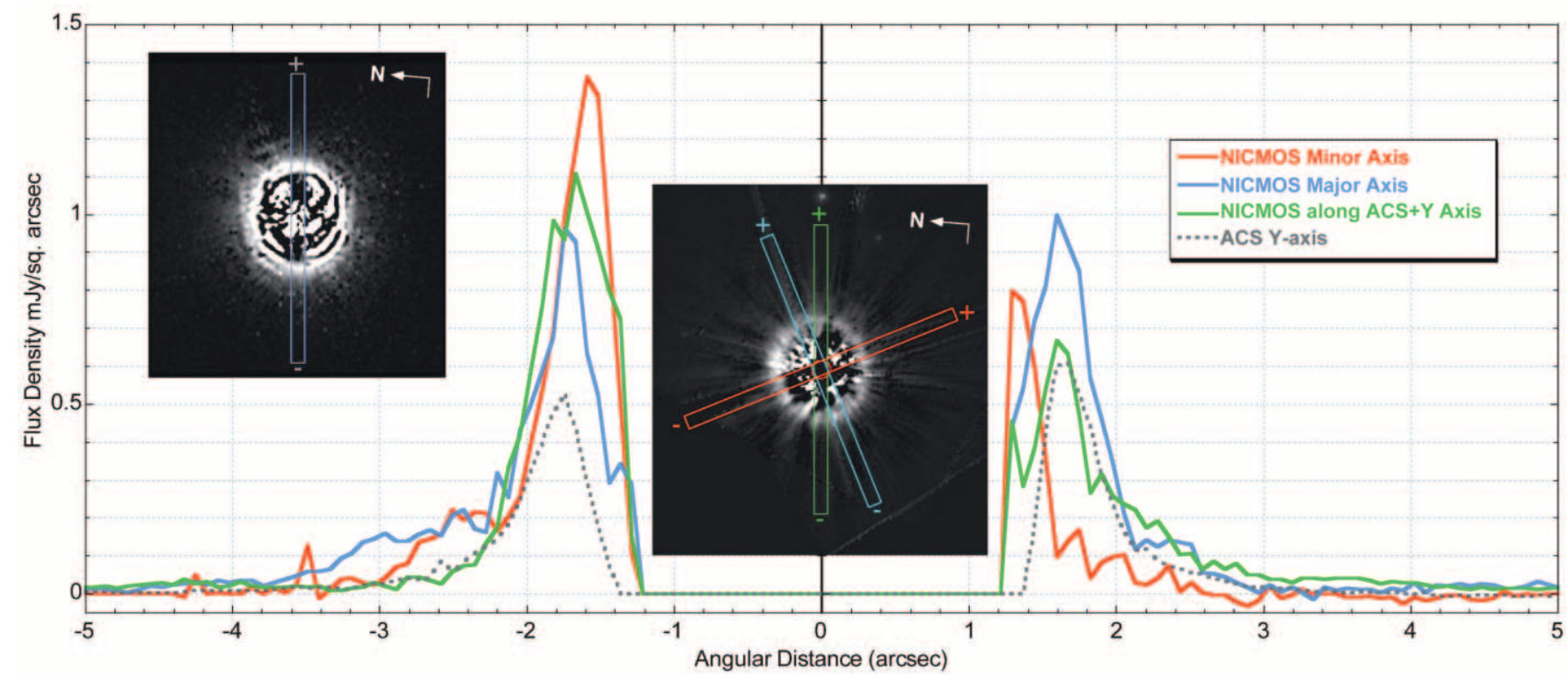

FIG. 6.- Radial SB profiles of the HD 181327 circumstellar ring. NICMOS measures along the disk minor and major axes and in the direction of the ACS image $+Y$-axis, in 0 "' 5 wide strips in radial increments of 1 pixel ( $~ 75.8$ mas). ACS measures are made identically along the HRC image $+Y$-axis (NICMOS and ACS images are displayed at the same spatial scale and celestial orientation). Uncertainties in the NICMOS measures may be estimated from the $1 \sigma$ errors shown in Fig. 8. Deriving the ACS measurement uncertainties accurately is problematic due to the very strong azimuthal dependence of the residuals. Based on the profile asymmetries, we estimate the uncertainties in the ACS measures in the 1 ". $5-2$ ". 2 radial region to be $< \pm 30 \%$ of the SB.

stellar flux density in the NICMOS F110W band is $5.9 \mathrm{Jy}$; hence, the fraction of starlight scattered by the disk, $f_{\text {scat }}$, at $r>1.2$ is $0.170 \% \pm 0.015 \%$ of the total stellar light. We include in our error estimation the uncertainty in our flux density measurement, and the absolute photometric calibration and transfer of the stellar flux density from $2 \mathrm{MASS} / J$ to NICMOS/F110W. This $1.1 \mu \mathrm{m}$ scattering fraction is roughly comparable to the $0.25 \%$ thermal IR excess found from the $\geq 24 \mu \mathrm{m}$ color-corrected IRAS and Spitzer flux densities $(\S 4)$.

The $1.1 \mu \mathrm{m}$ axial radial $S B$. - The SB was measured in radial strips along the disk major and minor axes, as well as in the direction along the image $Y$-axis in the orientation of the ACS HRC image (see Fig. 6) with radial increments of 75.8 mas (1 NICMOS camera 2 pixel) in both outward directions from the occulted star. At each radial increment the SB was determined from the median flux density measured in regions 1 pixel (along the radial) by 0.5 (6.5 pixels, corresponding to $\mathrm{a} \pm 10^{\circ}$ sector perpendicular to the radial at $\left.R_{\text {peak }}\right)$. The major-axis $\mathrm{SB}$ profile is symmetric about the star with a peak SB of $\sim 0.97 \mathrm{mJy} \operatorname{arcsec}^{-2}$ at $r=1$ ".71. In comparison, the asymmetric SB peaks along the minor axis on opposite sides of the star $\left(\sim 1.37\right.$ and $\left.0.79 \mathrm{mJy} \mathrm{arcsec}{ }^{-2}\right)$ are strongly indicative of directionally preferential scattering along the line of sight.

The $1.1 \mu \mathrm{m}$ deprojected azimuthal-median radial $S B .-\mathrm{We}$ measured the disk's $1.1 \mu \mathrm{m}$ SB profile as a function of distance from the star with elliptical aperture photometry. In doing so we assumed intrinsic circular symmetry and a disk inclination of $31^{\circ} .7$ from a face-on geometry. The measurement apertures were elliptical annuli of the same aspect ratio as the apparent disk ellipse (1:0.851) and co-aligned with the disk axes. The elliptical annuli were 75.8 mas wide on the major axis and were incremented in semimajor-axis length by the same amount. The deprojected radial SB profile medianed over all azimuth angles is shown in Figure 7. The radial SB was found to peak at all azimuths at isophotal radii of $86.3 \mathrm{AU}$ from the star (i.e., 1".706 if deprojected). The SB of the ring medianed over all azimuths at this peak radius was found to be $1.00 \pm 0.07 \mathrm{mJy}(\mathrm{F} 110 \mathrm{~W}=$ $15.62 \pm 0.07 \mathrm{mag}$ ) $\operatorname{arcsec}^{-2}$ (see Fig. 7), corresponding very closely to the ansal brightness of the ring on the major axis.

Ring width.-On the azimuthal median, the measured FWHM of the deprojected ring (see Fig. 7) is 0".538 ( 29 AU). At all azimuth angles the SB of the ring declines from the 1".706

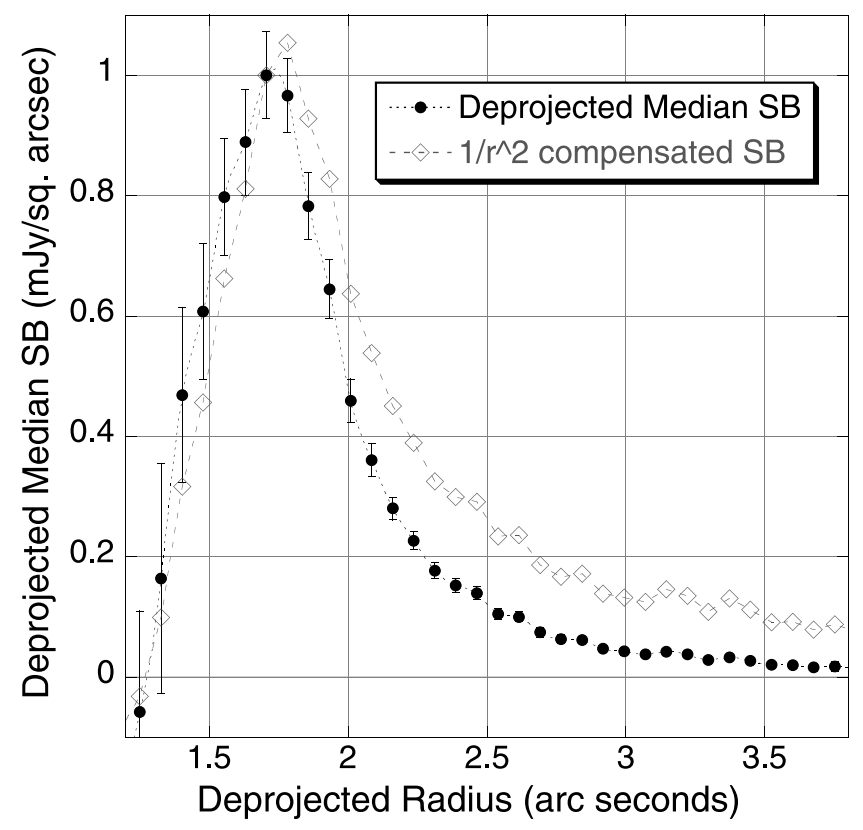

FIG. 7.- Azimuthally medianed $1.1 \mu$ m radial SB profile of the HD 181327 ring (deprojected from an inclination angle of $31^{\circ} .7$ from face-on) and the $1 \sigma$ uncertainties in each 75.8 pixel wide annular zone measured. The $1 \sigma$ error bars indicate the dispersion in the NICMOS SB measures around the ring at each elliptical annulus and do not include the $\sim 9 \%$ uncertainty in the absolute calibration of the SB. An adjusted profile compensating for the dilution of starlight with distance from the central star normalized to the radius of peak brightness is also shown. 
(deprojected) radius of peak brightness more steeply toward the interior. The $e^{-1} \mathrm{SB}$ falloff from the azimuthally medianed peak is 0 "'334 (16.9 AU) inwardly and 0 "'380 (19.2 AU) outwardly. In the region 1 .' $2<r<5$ ". 0 approximately $70 \%$ of the $9.6 \pm 0.8 \mathrm{mJy}$ scattered-light flux density at $1.1 \mu \mathrm{m}$ is contained within an annulus \pm 18 AU in width centered on the brightest zone at $r=$ $86 \mathrm{AU}$ from the star.

The $0.6 \mu \mathrm{m} \mathrm{SB}$. - Because of the strong azimuthal and radial dependencies in the ACS PSF subtraction residuals, SB profiles in the region of the ring were measured from the ACS HRC $0.6 \mu \mathrm{m}$ image only at $r>1$."4 in azimuthal sectors within $\pm 10^{\circ}$ of the image $Y$-axis. For reasons discussed in $\S 3.2$, these are the only sectors in this annular zone where the flux density of the disk could be measured with reliable error estimates against the azimuthally anisotropic background of diffractive PSF residuals. To permit a direct comparison to the NICMOS $1.1 \mu \mathrm{m}$ image, the ACS image was resampled to the NICMOS camera 2 pixel scale. The SB was measured in both directions from the occulted star along the ACS HRC image $Y$-axis in a manner identical to the equivalent NICMOS measures. The ACS HRC image $Y$-axis intersected the HD 181327 ring at $22^{\circ}$ from the disk major axis, as determined from the NICMOS image (see Fig. 6). The $0.6 \mu \mathrm{m}$ radial $\mathrm{SB}$ profile peaks at $\sim 1^{\prime \prime} .74$ (at P.A. $=95^{\circ}$ ) and $1^{\prime \prime} .66$ (at P.A. $\left.=275^{\circ}\right)$, with a mean SB of $0.57 \pm \lesssim 0.17 \mathrm{mJy} \operatorname{arcsec}^{-2}$ $\left(\mathrm{F} 606 \mathrm{~W}=16.9 \pm \lesssim 0.3 \mathrm{mag} \operatorname{arcsec}^{-2}\right.$ ), declining in intensity more rapidly inward than outward.

\subsection{Photometric Model of the $1.1 \mu \mathrm{m}$ Ringlike Disk}

Without consideration of the physical properties of the disk grains, we constructed a photometric model of the disk, predicated on the NICMOS $1.1 \mu \mathrm{m}$ image, to determine the width of the ring and the grain scattering efficiency as a function of azimuth angle (i.e., its scattering "phase function" in projection) and to test the measurement of its isophotal radius.

Form of the model. - We built a photometric model of the disk, assuming an inclined, intrinsically circularly symmetric ring. ${ }^{15}$ Based on the results of the isophotal ellipse fitting ( $\S 5.1$ ), we fixed the disk inclination to 31.7 and the orientation to $107^{\circ}$. Allowing for the $r^{-2}$ dilution of the central starlight, the SB of the ring was then parameterized by a set of four free parameters: $g_{\mathrm{HG}}$, $R_{\text {peak }}, \mathrm{SB}\left(\theta=0^{\circ}, R_{\text {peak }}\right)$, and $W$, as follows.

Azimuthal dependence.-We assumed a sky-plane-projected azimuthal angular $(\theta$, measured in the plane of the disk) dependence in the scattering efficiency $\left(g_{\mathrm{HG}}\right)$ of the disk grains with the line of sight to the scattering phase illumination angle, $\varphi=$ $\cos ^{-1}(\sin i \cos \theta)$. The spatially resolved deprojected surface brightness, $\mathrm{SB}(\theta, r)$, with $r$ measured in the plane of the inclined disk, is represented by a scattering phase function of the type suggested by Henyey \& Greenstein (1941):

$$
\mathrm{SB}(\theta, r)=\mathrm{SB}\left(0^{\circ}, R_{\text {peak }}\right)\left(\frac{1+g_{\mathrm{HG}}^{2}-2 g_{\mathrm{HG}} \sin i}{1+g_{\mathrm{HG}}^{2}-2 g_{\mathrm{HG}} \cos \varphi}\right)^{1.5}
$$

\footnotetext{
${ }^{15}$ We tested other functional forms, including model disks with uniform and radial power-law SB dependencies, but only the ring model provided a meaningful fit to the observational data. Both the uniform and radial power-law SB models "predicted" excessive levels of scattered light interior to the peak radius of the ring that was recovered with equal fidelity after model implantation into the target minus PSF and PSF minus PSF images; i.e., if the HD 181327 scattered-light ring exhibited such a form, dust-scattered light interior to the ring in excess of the declining levels actually detected (e.g., see Fig. 7) would have been observable (and to even smaller radii, due to more favorable image contrast) with statistical significance.
}

For the inclination of the HD 181327 disk, scattering phase angles in the range $58.3-121^{\circ} .7$ are explored.

Radial dependence. - We modeled a radial SB dependence with inward and outward exponential decays in SB from $R_{\text {peak }}$. We characterize the ring width, $W$, as the distance between the radial points where the $\mathrm{SB}$ at any azimuth, $\mathrm{SB}(\theta, r)$ declines to $\mathrm{SB}\left(\theta, R_{\text {peak }}\right) e^{-1}$, i.e., $36.8 \%$ of the peak $\mathrm{SB}$ at the same azimuth.

Convolution. - The disk models were convolved with the NICMOS camera 2 F110W point-source PSF for the SED of an F6 star using a noiseless, high-fidelity, model PSF generated with the HST TinyTim optical model ${ }^{16}$ (ver. 6.3) described by Krist \& Hook (1997).

Fitting process and region.-The set of parameters that best fits the observation for $i=31^{\circ} .7$, P.A. $=107^{\circ}$, was found by iterative $\chi^{2}$ minimization in observed minus model subtractions in the radial zone 1 ." $2<r<3$ ". 0 . The PSF subtraction residuals in the final image have complex spatial characteristics that increase in intensity with decreasing angular distance from the coronagraphically occulted star. Their amplitudes set the inner distance into which these observations can effectively probe. In the fitting process we exclude data for which $r<1$ '.2, as this region conveys little (or no) meaningful information.

At angular distances $>1$ ". 2 , the instrumentally induced PSF subtraction residuals are typically about a pixel in azimuthal extent, but are correlated on much larger spatial scales radially. These PSF "tendrils" impede the visibility of azimuthal variations in the ring SB on comparable spatial scales. To illustrate the underlying form of the ring, we removed these PSF subtraction residuals by applying an elliptical low-pass filter to the image of the ring (Fig. 2c). We used $\mathrm{a} \pm 9^{\circ}$ rotational boxcar kernel of width 3.5 pixels (i.e., 2.5 resolution elements at the radius suggested by isophote fitting), so only structures with approximately half that width are passed. Any "real" features of comparable azimuthal spatial frequencies are indistinguishable from PSF subtraction artifacts and also smoothed in this process. These high angular spatial frequency features are removed from the filtered disk image at the expense of a degradation in azimuthal spatial resolution. The two bright spots in the northeastern sector of the ring (displayed as "hard white" in Fig. 2c) coincide with the location of the wider $H S T+$ NICMOS diffraction spikes in two of the four original (precombined) PSF-subtracted images. These regions of enhanced brightness likely arise from larger spatial scale, but localized, imperfections in the PSF subtractions and are not intrinsic to the disk. For this reason, in the fitting process we also exclude these two regions in the northeastern quadrant of the ring, due to the likelihood of their arising from large spatial scale artifacts in the PSF-subtracted image.

\subsection{Model-fitting Results}

Peak radius. - The peak radius $R_{\text {peak }}$ was found to be $22.5 \pm$ 0.4 pixels, in agreement with the radius found from fitting ring isophotes. This corresponds to a physical radius of $86.3 \pm 3.9 \mathrm{AU}$.

Characteristic width. - The characteristic width $W$ was found to be $8.26 \pm 0.9$ pixels. In this characterization, $69.2 \%$ of the light scattered by the dust originates in a $35.8 \pm 4.0$ AU wide annulus centered $86.3 \pm 3.9$ AU from the star. (The $4.1 \%$ uncertainty in the distance to HD 181327 from its parallax measure has been added in quadrature to the formal fitting error in expressing the uncertainty in $R_{\text {peak }}$ and $W$ in AU.)

The $W=35.8 \pm 4.0$ AU fit to the ring width (as characterized) is in very good agreement with the estimate derived from

\footnotetext{
16 See http://www.stsci.edu/software/tinytim/tinytim.html.
} 

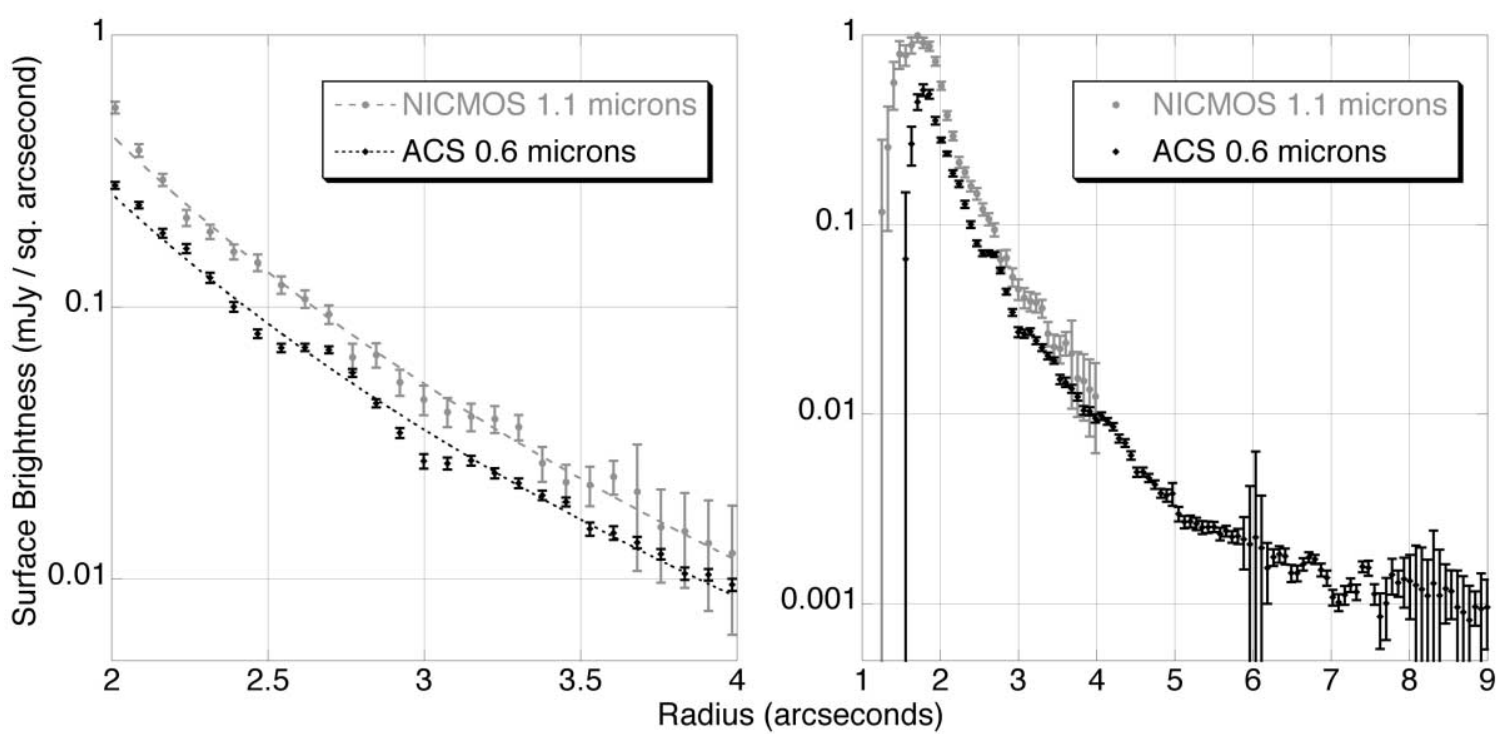

FIG. 8. - NICMOS and ACS deprojected SB profiles of the HD 181327 circumstellar disk from (left) $2^{\prime \prime}-4^{\prime \prime}(\sim 100-200$ AU) and (right) inner detection radii to $9^{\prime \prime}(\sim 450 \mathrm{AU})$. NICMOS profile is limited to $r<4^{\prime \prime}$ due to field of view truncation (see Fig. 9) and integration depth. The $1 \sigma$ error bars are the standard deviations of the measured SB in each 1 pixel wide radial zone about the zonal median and do not include uncertainties in the absolute calibration of the disk flux densities. The ACS profile was measured after resampling the image to the NICMOS pixel scale (effectively smoothing the image with an $\approx 3$ pixel $\times 3$ pixel kernel).

the direct measures of the median radial profile of the deprojected disk. Neither changes significantly with azimuth when measured in $9^{\circ}$ wide azimuthal sectors (in the absence of subtraction artifacts) around the ring. The large-scale azimuthal uniformity of the $\mathrm{FWHM}_{\text {ring }}$ suggests a constant product of the optical depth and grain albedo $(\tau \omega)$ with azimuth, and perhaps then azimuthally undifferentiated grains.

Scattering phase function.-The HD 181327 ring exhibits strong directionally preferential scattering with maxima and minima along the line of sight, i.e., in the direction of the ring minor axis, with $g_{\mathrm{HG}}=0.30 \pm 0.03$, as found in the fitting process. (Discrete SB measurements along small azimuthal sectors at constant deprojected radii depart from this fit due to biasing by local PSF subtraction residuals. ${ }^{17}$ ) A photometric model of the disk, convolved with the NICMOS camera $2 \mathrm{PSF}, g_{\mathrm{HG}}=0.30$, and $i=$ 31.7 from face-on (shown in Fig. 2b), predicts ansal SBs of $0.863 \mathrm{mJy} \operatorname{arcsec}^{-2}$ and minor-axis SB peaks of 1.37 and $0.56 \mathrm{mJy}$ $\operatorname{arcsec}^{-2}$, each in agreement with the photometric measures (Fig. 6) within the $1 \sigma$ measurement uncertainties assessed with respect to the azimuthal median.

\section{6. $\mathrm{NICMOS}+\mathrm{ACS}$}

The NICMOS and ACS instruments each better probed a different spatial regime of the HD 181327 circumstellar disk (for a comparative overview of the $H S T$ coronagraphs, see Schneider et al. 2003). ${ }^{18}$ While the spatial domain of overlap with high

17 The SB of the disk at $R_{\text {peak }}$ was also discretely measured as a function of elliptical azimuth around the ring. Measurements were made in "face-on projection," with the disk SB preserved in the geometric transformation. Circular photometric apertures of both 3.5 and 7 pixel diameters ( 1 and 2 times the FWHM of the SB peak) were used to measure the SB every $9^{\circ}$ ( 40 measurements), and every $18^{\circ}$ (20 measurements) around the ring, respectively. Aperture dilution of the nonuniform flux density distribution is less severe in discrete measurements with the smaller aperture, but these individual measures are more influenced by local subtraction residuals than the larger aperture. The SB measures using both apertures were in agreement with a $g_{\mathrm{HG}}=0.30 \pm 0.03$ scattering phase function.

18 See http://nicmosis.as.arizona.edu:8000/POSTERS/AAS_JAN2004_CORON .jpg. photometric fidelity in the radial region of the debris ring is small, for the reasons noted in $\S 3.2$, the NICMOS and ACS observations together inform us further on the global structure and properties of the disk and its environment. The NICMOS imagery reveals the detailed structure of the disk and the region interior to $\sim 3^{\prime \prime}$, whereas the larger ACS field and deeper integrations reveal the faint outer nebulosity surrounding the ring at $r>4^{\prime \prime}$. In the regions commonly sampled the nearly factor of 2 wavelength coverage afforded by the ACS/F606W $(0.6 \mu \mathrm{m})$ and NICMOS/F110W $(1.1 \mu \mathrm{m})$ filters provide rudimentary color information on the disk grains.

\section{1. $[\mathrm{F} 606 \mathrm{~W}]-[\mathrm{F} 110 \mathrm{~W}]$ Color of the Disk at $R_{\text {peak }}$}

HD 181327 has a $V-J$ (similar to $[\mathrm{F} 606 \mathrm{~W}]-[\mathrm{F} 110 \mathrm{~W}]$ for an F5/F6 V star) color index of $\sim+0.80$. As noted in $\S 5.2$, the $0.6 \mu \mathrm{m} \mathrm{SB}$ of the disk could be measured reliably only in small regions along the debris ring between 1".4 and 2".0 flanking the ACS HRC image $Y$-axis (indicated in Fig. $3 a$ ) at celestial position angles of $95^{\circ}$ and $275^{\circ}, 22^{\circ}$ from the disk major axis (as shown in Fig. 6). Identical measures from the best-fit $1.1 \mu \mathrm{m}$ scattered-light model of the ring based on the NICMOS image found SBs of 1.30 and $0.72 \mathrm{mJy} \operatorname{arcsec}^{-2}$ at the same position angles, respectively. The mean $1.1 \mu \mathrm{m} \mathrm{SB}$ in these regions corresponds to $15.61 \pm 0.08\left(\mathrm{~F} 110 \mathrm{~W}_{\mathrm{mag}} \operatorname{arcsec}^{-2}\right)$. Hence, in these regions where the disk-scattered light peaks radially, the disk grains appear significantly red, with a $[\mathrm{F} 606 \mathrm{~W}]-[\mathrm{F} 110 \mathrm{~W}]$ color index of $+1.32 \pm 0.3$. Correcting for the color of the star, the disk grains in this region have an intrinsic $[\mathrm{F} 606 \mathrm{~W}]-[\mathrm{F} 110 \mathrm{~W}]$ color index of $+0.5 \pm 0.3$.

\subsection{The Outer Regions of the Disk}

Beyond $R_{\text {peak }}$, in the region of high photometric reliability of $100-200 \mathrm{AU}\left(r \lesssim 4^{\prime \prime}\right.$ in the deprojected NICMOS image), the $1.1 \mu \mathrm{m} \mathrm{SB}$ declines as $15.75 r^{-5.2} \mathrm{mJy} \operatorname{arcsec}^{-2}$ on the azimuthal median (see Fig. 8). Using the ring inclination and orientation derived from the NICMOS image, we similarly deproject the ACS 


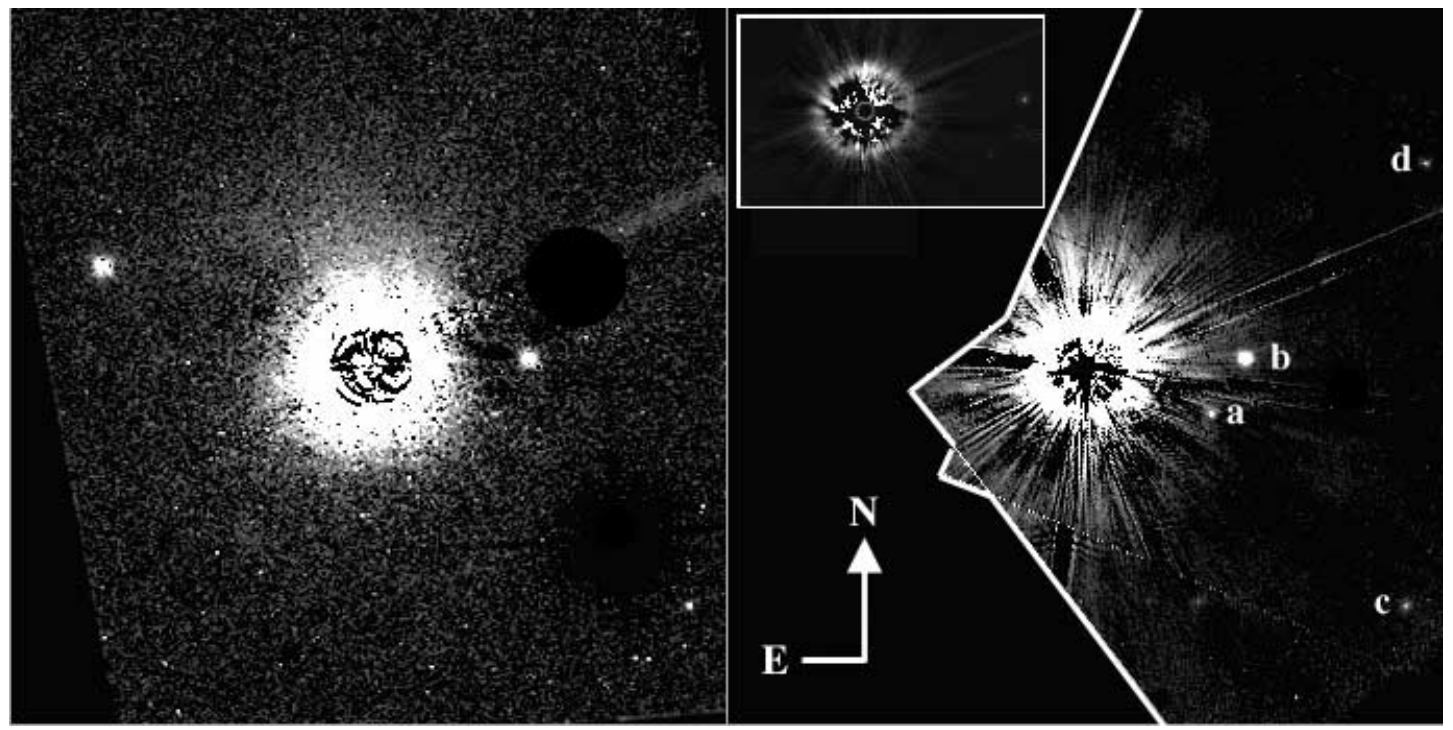

FIG. 9.- Outer regions of the HD 181327 disk in (left) the ACS and (right) the NICMOS PSF-subtracted images within \pm 12 ". 5 of the central star. Both images are square root displays with maximum intensities (hard white) corresponding to 1.4 instrumental counts $\mathrm{s}^{-1}$ pixel ${ }^{-1}$. Four stars common to both fields are noted (denoted a-d). Inset: Debris ring revealed in the NICMOS image, shown at the same spatial scale and image orientation (linear display from-0.25 to +10 counts $\mathrm{s}^{-1}$ pixel $\left.^{-1}\right)$.

image and find a very similar power-law index with a monotonic decline in the $0.6 \mu \mathrm{m} \mathrm{SB}$ of $7.98 r^{-4.9} \mathrm{mJy} \mathrm{arcsec}^{-2}$ in the same region (also shown in Fig. 8). The azimuthally medianed $[\mathrm{F} 606 \mathrm{~W}]-[\mathrm{F} 110 \mathrm{~W}]$ color index of the disk throughout the $100-$ 200 AU region is statistically identical to that found at the two measurable locations at $86 \mathrm{AU}(\S 5.2)$. Hence, from these limited data we see no evidence of radial differentiation in the color of the disk grains.

The ACS image reveals a diffuse low-SB nebulosity to a distance of $\lesssim 9^{\prime \prime}$ from the star (Fig. 9, left), the inner portion (beyond $\sim 4^{\prime \prime}$ ) of which is only hinted at in the NICMOS image (Fig. 9, right). The nebulosity seen in the ACS image, with a mean $0.6 \mu \mathrm{m} \mathrm{SB}$ in the $4^{\prime \prime}<r<9^{\prime \prime}$ region of $\approx 21.5$ $\left(\mathrm{F} 606 \mathrm{~W}_{\mathrm{mag}}\right) \operatorname{arcsec}^{-2}$, is azimuthally asymmetric, with the brightest part in an $\sim 90^{\circ}$ sector to the north of the star and "curving" in the outer regions toward the east. HST coronagraphic observations have revealed spiral structures in the circumstellar disks of the Herbig Ae/Be stars HD 100546 (Grady et al. 2001) and HD 141569A (Clampin et al. 2003). In the latter case, Clampin et al. postulated that HD 141569's outer "arms" might have arisen from tidal interactions from a passing star, which could also be the causal mechanism for both the disk's stellar photocentric offset and its large azimuthal surface density variations. Neither of these phenomena is seen in the HD 181327 disk. The southwest arm of the HD 141569A disk seems to be associated with its M-star companions, but no tidally interacting companions have been found for HD 181327 (see $\S 6.3$ ). Kalas et al. (2002) have reported on the existence of Pleiades-like reflection nebulosities in the environments of Vega-like stars of spectral types K2 V-B9.5 V (i.e., similar to HD 181327), although an examination of Spitzer 24 and $70 \mu \mathrm{m}$ images of the field around HD $181327(\S 4)$ does not reveal any emission structure that could be identified with the low-SB feature seen in the ACS $0.6 \mu \mathrm{m}$ image. Hence, the observations we report here, unfortunately, do not inform us on the physical nature of the axially asymmetric nebular structure in proximity to HD 181327 that may or may not be physically associated with its disk.

\subsection{Tests for Companionship}

In $\oint 1$ we suggest that HD 181327 and the 10-30 Myr star HR 7329 (Lowrance et al. 2000) are a common proper motion (PM) pair, strengthening the argument for HD 181327's youth. Four pointlike objects (stars) are seen in the more immediate vicinity of HD 181327 in both the NICMOS and ACS images (denoted a-d in Fig. 9). Although stars a and b were within the 12 ". $6 \times 12$ ". 6 SHARP-I (Neuhäuser et al. 2003) and 12 ".7 $\times 12$ ".7 SHARP-II (Chauvin et al. 2003) survey fields, neither was detected in either study. We also searched for low-luminosity (substellar) companion candidates in close angular proximity to HD 181327 using the NICMOS coronagraphic images. Following Schneider \& Silverstone (2003b) and Lowrance et al. (2005), we implanted model "PSF stars" (using noiseless TinyTim 6.3 PSFs; Krist \& Hook 1997) into a difference image made of the two differentially oriented coronagraphic images of HD 181327. Even in the presence of the HD 181327 disk, at and interior to the radius of peak SB, we found (similar to Weinberger et al. [1999] in the case of HD 141569A) that at this approximate age brown dwarf or giantplanet companions with masses $\gtrsim 10 M_{\text {Jup }}$ would be detectable to an interior radius of $\approx 1^{\prime \prime}$. To these approximate sensitivity limits, no close-proximity substellar companion candidates were found.

The age of HD 181327, presumed $\sim 12 \mathrm{Myr}$ as a member of the $\beta$ Pictoris moving group, could be further constrained by estimating the age of a low-mass (stellar) companion (e.g., by the presence of $\mathrm{Li}$, the $\mathrm{H} \alpha$ equivalent width, X-ray activity, etc.). We tested the possibility of companionship of the four stellar-like objects appearing in the NICMOS and ACS images through differential PM measures over the $1.5 \mathrm{yr}$ between observations. On the aggregate, the four had measured differential PMs of $(\Delta$ R.A., $\Delta$ decl. $)=(-42.3,+83.9)$ mas $\mathrm{yr}^{-1}$ with a $1 \sigma$ disper-

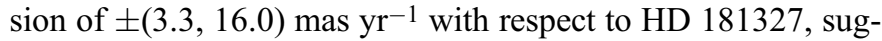
gestively indicating the reflective motion of HD 181327 [the largest outlier, star c, was discrepant from the ensemble mean by

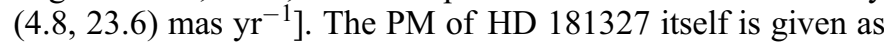
$(+24,-82)$ mas $\mathrm{yr}^{-1}$ by Perryman et al. (1997). Given the very good internal agreement in the measured PM of the four stars, the 


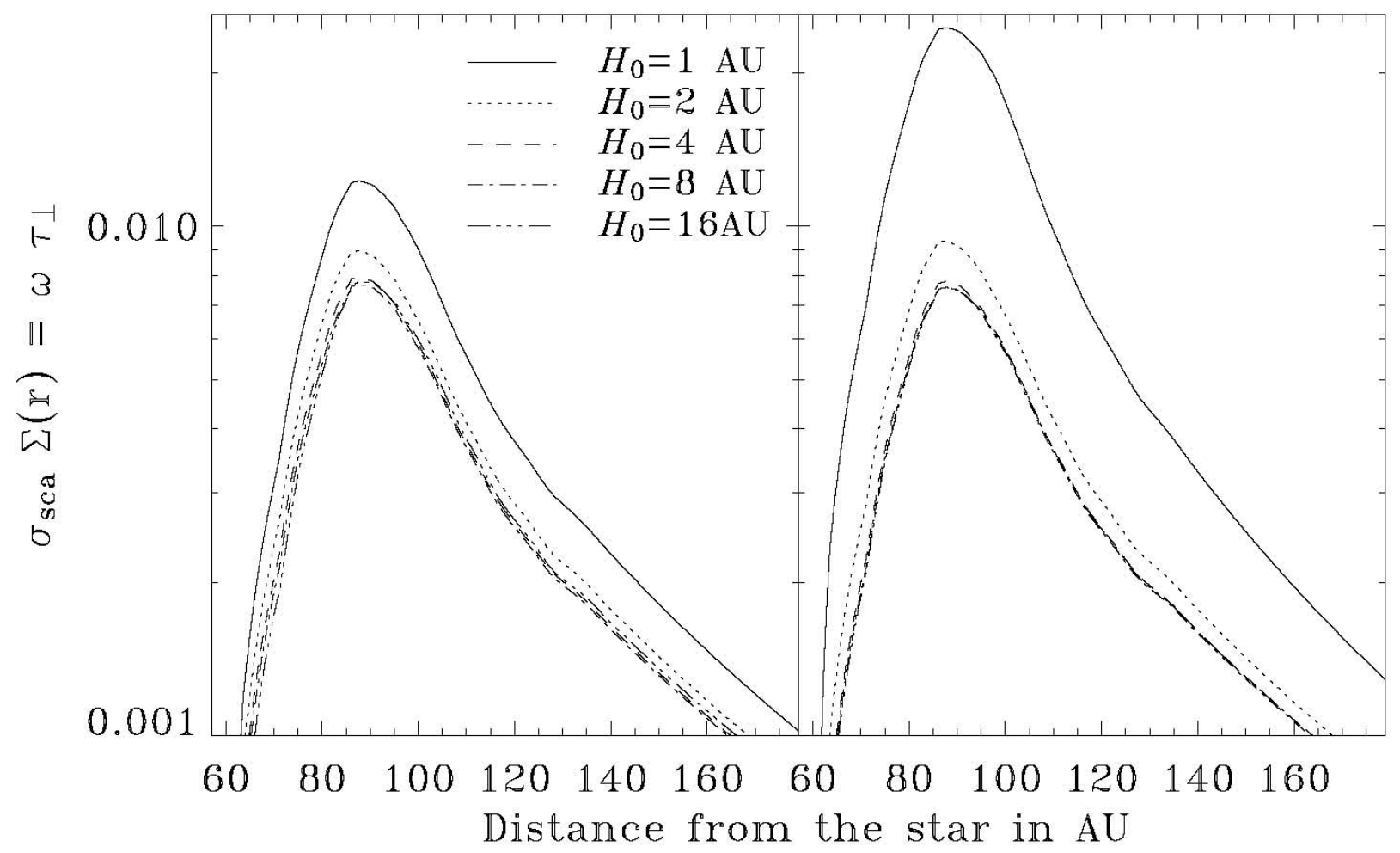

Fig. 10.- Surface density profiles multiplied by the mean scattering cross section at $1.1 \mu \mathrm{m}$, assuming different vertical scale heights $\left(H_{0}\right)$. Left, exponential vertical profile $(\gamma=1)$; right, Gaussian profile $(\gamma=2)$.

dispersion being $<(0.14,0.20) \sigma$ of the PM of HD 181327, all four, unfortunately, are very likely background stars. ${ }^{19}$

\section{DISK/GRAIN PROPERTIES}

\subsection{Inversion of the Scattered-Light SB Profile: Approach}

We modeled the scattered-light surface brightness, $\mathrm{SB}(x)$, of an axisymmetric, inclined, and optically thin disk, measured at a projected distance $x$ from the star along the apparent major axis, following the methodology described by Augereau \& Beust (2006) such that

$\frac{\mathrm{SB}(x)}{\Phi^{*}}=\int_{x}^{r_{\max }} \sigma_{\mathrm{sca}} \rho(r, z) \frac{f(\varphi)+f(\pi-\varphi)}{r^{2}-x^{2} \cos i} \frac{\sin i}{\sqrt{r^{2}-x^{2}}} r d r$,

where $i$ is the disk inclination from pole-on, $\rho(r, z)$ is the disk number density in the cylindrical frame centered on the star and attached to the disk, $r_{\text {max }}$ is the outer disk radius, $\Phi^{*}$ is the stellar flux at the Earth at the wavelength considered, and $\sigma_{\text {sca }}$ and $f(\varphi)$ are the scattering cross section of the grains and the anisotropic

19 The magnitude of the apparent discrepancy in the reflective PM in right ascension of HD 181327 over the $1.5 \mathrm{yr}$ between the observations, assessed from the four star ensemble compared to its a priori measured value, is $\sim 28$ mas (approximately $1 / 3$ of a NICMOS camera 2 pixel). We attribute this discrepancy to an (unresolved) intercamera differential astrometric systematic uncertainty, likely due to differential camera distortion corrections and/or occulted target centration errors (as previously noted in $\S 3.2$ for the ACS observation). We note that all four "reference stars" were spatially distributed within a single quadrant (to the west of HD 181327) due, in part, to the intersecting areas of the two camera fields. A more isotropic distribution of reference stars (or a two-epoch observation with the same instrument) would have provided a better absolute astrometric solution. A fifth, brighter, star appears in the ACS field (to the east of HD 181327) only, so its differential PM could not be measured. scattering phase function at phase angle $\varphi$, respectively, both averaged over the grain size distribution.

In the case of HD 181327, the mean phase function $f(\varphi)$ is observationally constrained and can be approximated by a HenyeyGreenstein type phase function with $g_{\mathrm{HG}}=0.3 \pm 0.03$ at $1.1 \mu \mathrm{m}$, as noted in $\S 5.4$. We also presume an axisymmetric disk predicated on the observed bilateral symmetry of the scattered-light ring in the NICMOS image. Therefore, for a given vertical dust distribution, $Z(r, z)$, the product of the midplane number density, $\rho(r, 0)=\rho(r, z) / Z(r, z)$, and the mean scattering cross section, $\sigma_{\text {sca }}$, can be obtained by directly inverting the observed SB profile $\mathrm{SB}(x)$. We do so using the technique detailed in Augereau \& Beust (2006) and discuss the properties of the dust ring as a function of $Z(r, z)$.

We characterize the vertical distribution with a three-parameter function $Z(r, z)=\exp \left\{-[|z| / H(r)]^{\gamma}\right\}$, where $H=H_{0}\left(r / r_{0}\right)^{\beta}$ is the vertical scale height and $r_{0}$ is the observed radius of the ring (86.3 AU). We calculated the midplane number density for both exponential $(\gamma=1)$ and Gaussian $(\gamma=2)$ profiles, assuming either a linearly flaring $(\beta=1)$ or nearly flat $(\beta=0.1)$ disk. In each case, 20 scale heights, $1 \mathrm{AU} \leq H_{0} \leq 20$ AU, were considered, corresponding to $0.012 \lesssim H_{0} / r_{0} \lesssim 0.23$ (see $\S 7.3$ ).

To compare the profiles, we introduce the disk surface density, $\Sigma(r)$, which can be written

$$
\begin{aligned}
\sigma_{\mathrm{sca}} \Sigma(r) & =\int_{-\infty}^{+\infty} \sigma_{\mathrm{sca}} \rho(r, z) d z \\
& =C_{\gamma} H(r) \sigma_{\mathrm{sca}} \rho(r, 0)=\omega \tau_{\perp}(r),
\end{aligned}
$$

where $\tau_{\perp}(r)$ is the vertical optical thickness at $1.1 \mu \mathrm{m}, \omega$ is the mean albedo in the NICMOS F110W passband averaged over the grain size distribution, and $C_{\gamma}$ is a constant value depending on $\gamma\left(C_{1}=2\right.$ and $\left.C_{2}=\sqrt{\pi}\right)$. 


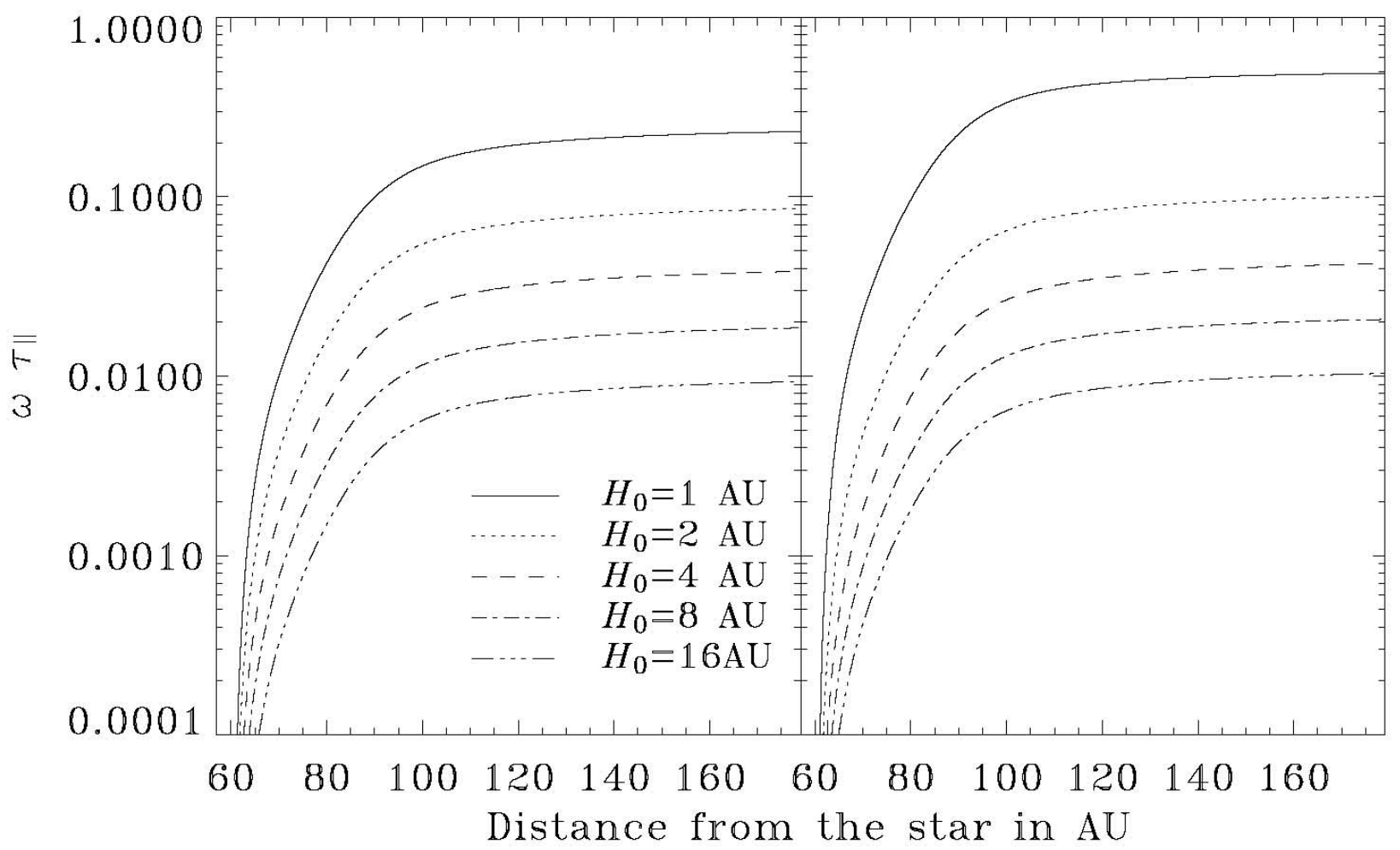

FIG. 11. - Midplane disk thickness in the NICMOS F110W band as a function of the distance from the star for different vertical scale heights $\left(H_{0}\right)$. Left, Exponential vertical profile $(\gamma=1)$; right, Gaussian profile $(\gamma=2)$.

\subsection{Inversion of the Scattered-Light SB Profile: Results}

Figure 10 shows several $\sigma_{\text {sca }} \Sigma(r)=\omega \tau_{\perp}(r)$ profiles obtained by directly inverting the averaged NICMOS F110W SB profile. For sufficiently large values of $H_{0}$, the vertical optical thickness at $1.1 \mu \mathrm{m}$ is independent of the shape of $Z(r, z)$, while the width of the ring ${ }^{20}$ of $43 \pm 1 \mathrm{AU}(\mathrm{FWHM} \simeq 32.5 \pm 0.5 \mathrm{AU})$ depends slightly on the vertical profile. At large distances, the surface density profile falls off as $\sim r^{-3}$, in agreement with the observed $r^{-5}$ SB profile. A surface density profile $\Sigma(r) \propto r^{-3}$ is fully consistent with dust grains supplied by parent bodies on circular orbits and placed in orbits of high eccentricity by a drag force $\propto a^{2} /\left(r^{2}+\right.$ $z^{2}$ ), where $a$ is the grain size (e.g., see eq. [3] and Fig. 1 of Lecavelier des Étangs et al. 1996). This model also requires a differential grain size distribution that does not depart too much from a $a^{-3.5}$ power law and is in good agreement with the conclusions from the SED fitting (see $\S 8$ ). Moreover, because the model needs the dust dynamics to be largely unaffected by gas, the shape of the observed dust profile may indicate low gas density, as it has been similarly shown for $\beta$ Pictoris (Thébault \& Augereau 2005).

As HD 181327 is a star of spectral type F5/F6 V, radiation pressure contributes at least partly to the total drag force on the grains. In that picture, the dust particles observed at distances larger than $\sim 100 \mathrm{AU}$ most likely originate from a belt of large solid bodies peaking close to $87.6 \mathrm{AU}$.

The midplane optical thickness, $\tau_{\|}$, in the NICMOS F110W passband is related to the vertical thickness through

$$
\omega \tau_{\|}(r)=\int_{0}^{r} \sigma_{\text {sca }} \rho(R, 0) d R=\int_{0}^{r} \frac{\omega \tau_{\perp}(R)}{C_{\gamma} H(R)} d R,
$$

\footnotetext{
${ }^{20}$ Full width at $e^{-1}$ of the maximum.
}

where $\tau_{\|}$is the $1.1 \mu \mathrm{m}$ optical thickness calculated from the region between the inner edge of the disk and a distance $r$. As shown in Figure 11, with $H_{0}$ too small the disk appears marginally optically thick in the midplane. Using the constraints on the grains for astronomical silicates (Weingartner \& Draine 2001; $\S 8$ ), we estimate the mean albedo, $\omega$, to be $\approx 0.6$. Although the albedo is not well constrained, we can safely conclude that $H_{0}$ must be $\gtrsim 4 \mathrm{AU}\left(H_{0} / r_{0}>0.05\right)$, to ensure that the disk is optically thin in all directions at $1.1 \mu \mathrm{m}$. When this condition is fulfilled, the ring vertical thickness, $\tau_{\perp}(r)$, reaches a maximum of $\sim 0.01$ (assuming $\omega \approx 0.6$ ) at $87.6 \mathrm{AU}$ (Fig. 10). Assuming a grain mass density of $3.5 \mathrm{~g} \mathrm{~cm}^{-3}$ and a $a^{-3.7}$ differential grain size distribution between $1 \mu \mathrm{m}$ and $1 \mathrm{~mm}$, we infer a total dust mass of $\approx 4.1 M_{\text {Moon }}$ $\left(\approx 0.05 M_{\oplus}\right)$.

The collision timescale may be estimated as suggested by Backman \& Paresce (1993),

$$
t_{\mathrm{coll}} \simeq \frac{\sigma_{\mathrm{sca}}}{\pi\left\langle a^{2}\right\rangle} \frac{r^{1.5}}{2 \omega \tau_{\perp}(r) \sqrt{G M}},
$$

where $\pi\left\langle a^{2}\right\rangle$ is the mean geometric cross section of the grains averaged over the grain size distribution, $G$ is the gravitational constant, and $M$ is the stellar mass $\left(\sim 1.35 M_{\odot}\right.$ for an F5/F6 V star). The size distribution derived in $\S 8$ gives $\sigma_{\text {sca }} / \pi\left\langle a^{2}\right\rangle \simeq 1.4$. For such grains located within $150 \mathrm{AU}$ of the star, the collision timescale is $<10^{5} \mathrm{yr}$, except very close to the inner ring edge, where the density is extremely low. At the position of the peak surface density, $t_{\text {coll }} \approx 10^{4} \mathrm{yr}$ (hence about 3 orders of magnitude smaller than the star's age). The ring is then collision dominated, and the smallest of the released grains can populate the outer disk, thanks to radiation pressure commensurate with the $r^{-3}$ surface density profile at large distances. The short collision timescale implies that any dynamical process for the dust grains lasting longer than $10^{4}-10^{5} \mathrm{yr}$ can thus be neglected. This is the 


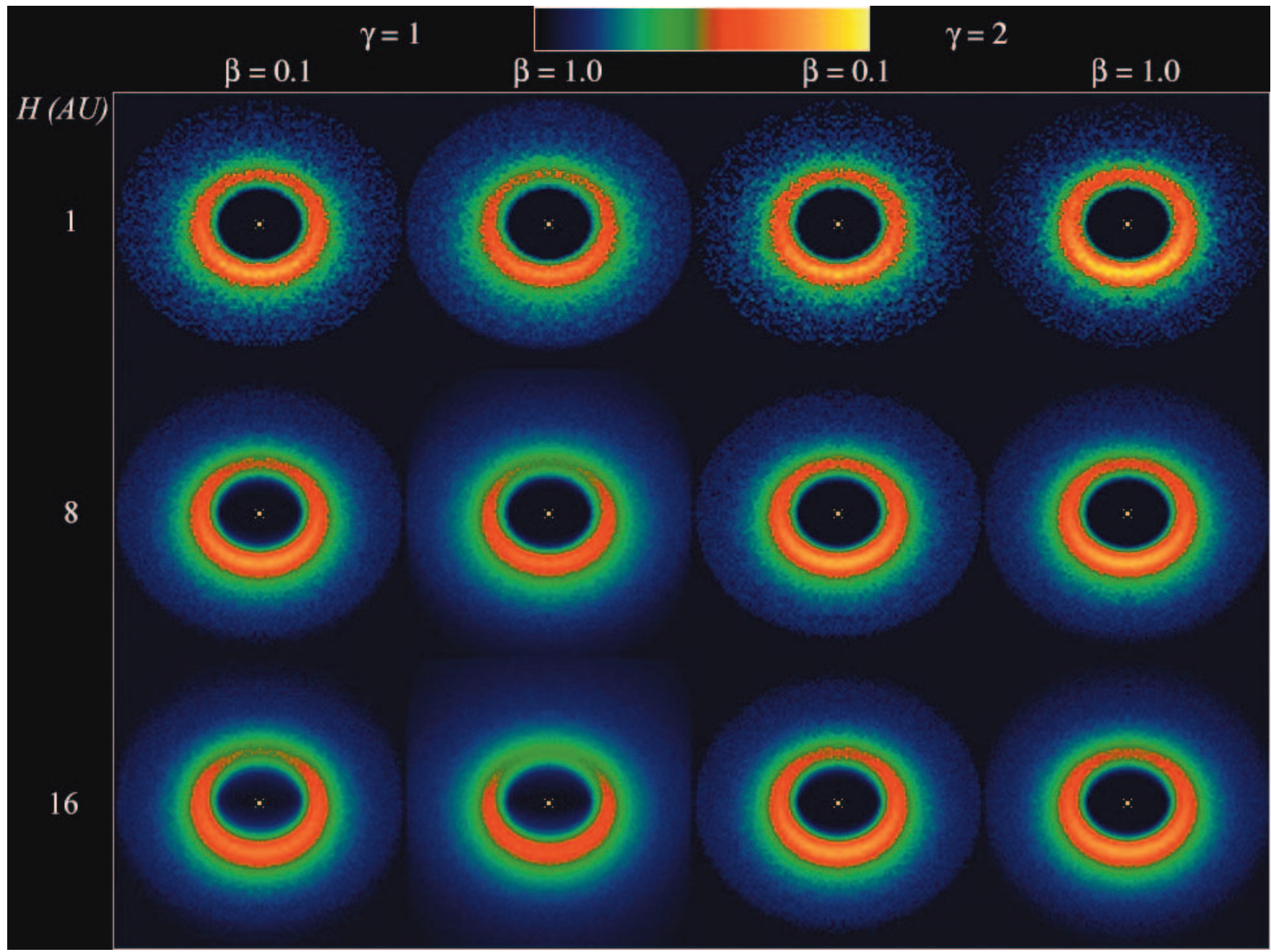

FIG. 12. - Scattered-light patterns from representative nearly flat $(\beta=0.1)$ and linearly flaring $(\beta=1)$ model disks with both exponential $(\gamma=1)$ and Gaussian $(\gamma=2)$ midplane number density profiles for disks with vertical scale heights, $H_{0}$, of 1,8 , and $16 \mathrm{AU}$ at $r_{0}\left(r_{0}=86.3 \mathrm{AU}\right.$ for HD 181327). Square root stretched image displays are normalized to the peak intensity of the $\left(\beta=1, \gamma=2, H_{0}=1 \mathrm{AU}\right)$ model disk (upper right panel).

case of Poynting-Robertson drag for which the depletion timescale at 87.6 AU for just-bound grains compares to the stellar age (i.e., $\sim 10 \mathrm{Myr}$ ); i.e., dust cannot populate the disk interior to the ring due to Poynting-Robertson drag.

\subsection{Scattered-Light Models and Fits}

Synthetic scattered-light images of the HD 181327 circumstellar disk were generated employing the three-dimensional radiative transfer code, MCFOST, described by Pinte et al. (2006). The product of density, $\rho(r, z)$, and scattering cross section, $\sigma_{\text {sca }}$, resulting from the inversion of the SB profiles ( $\S \S 7.1$ and 7.2) were used assuming a Henyey-Greenstein scattering phase function with $g_{\mathrm{HG}}=0.3(\S 5.4)$. Photon packages escaping the system with inclination angles between $30^{\circ} .5$ and $32^{\circ} .5$ were recorded in the synthetic images. Representative model disks for a subset of characterizing parameters $\alpha, \beta$, and $H_{0}$, corresponding to several of the profiles discussed in $\S 7.2$ (and presented in Figs. 10 and 11), are shown in Figure 12.

The spatially resolved flux densities predicted by the MCFOST scattered-light models were fit to the NICMOS image in the annulus of high $\mathrm{S} / \mathrm{N}(r \approx 1.3-2$."3) enclosing the observed scatteredlight ring. Each of the previously discussed models, tiling the parameter spaces illustrated in Figure 12, were fit to the NICMOS image by $\chi^{2}$ minimization, scaling the intensities of the predicted scattered-light patterns to the observed spatially resolved flux densities. A representative example (the $\beta=1, \gamma=2, H_{0}=1 \mathrm{AU}$ case) and its fit to the NICMOS image after $\chi^{2}$ minimization are shown in Figure 13. Within each $(\beta, \gamma)$ family of models, the reduced $\chi^{2}$ estimation was relatively flat for $H_{0}<8$ AU. In $\S 7.2$ we separately concluded that $H_{0}$ must be $\gtrsim 4 \mathrm{AU}\left(H_{0} / r_{0}>0.05\right)$; hence we suggest $4 \mathrm{AU} \lesssim H_{0} \lesssim 8 \mathrm{AU}$.

\section{GRAIN SIZE DISTRIBUTION}

Both the ACS $(0.6 \mu \mathrm{m})$-NICMOS $(1.1 \mu \mathrm{m})$ color of the HD 181327 disk and the measured scattering asymmetry factor $\left(g_{\mathrm{HG}}=0.3\right.$ at $\left.1.1 \mu \mathrm{m}\right)$ indicate that the scattered-light images are dominated by submicron grains. The grain properties that may explain the scattered-light observations should also be consistent with other independent constraints for the grains. In particular, grains at the observed radius of 86.3 AU should produce an infrared signature consistent with the measured long-wavelength excesses, and the grain properties should be consistent with basic dynamical considerations. Because HD 181327 is an F5-F6 type star, the radiation pressure force on the grains is expected to be significant and can expel sufficiently small grains from the disk on very short timescales.

We have assumed a unity-normalized differential grain size distribution $\left[\int_{a_{\min }}^{a_{\max }} d n(a)=1\right]$ of the form $d n(a) \propto a^{\kappa} d a$ between a 


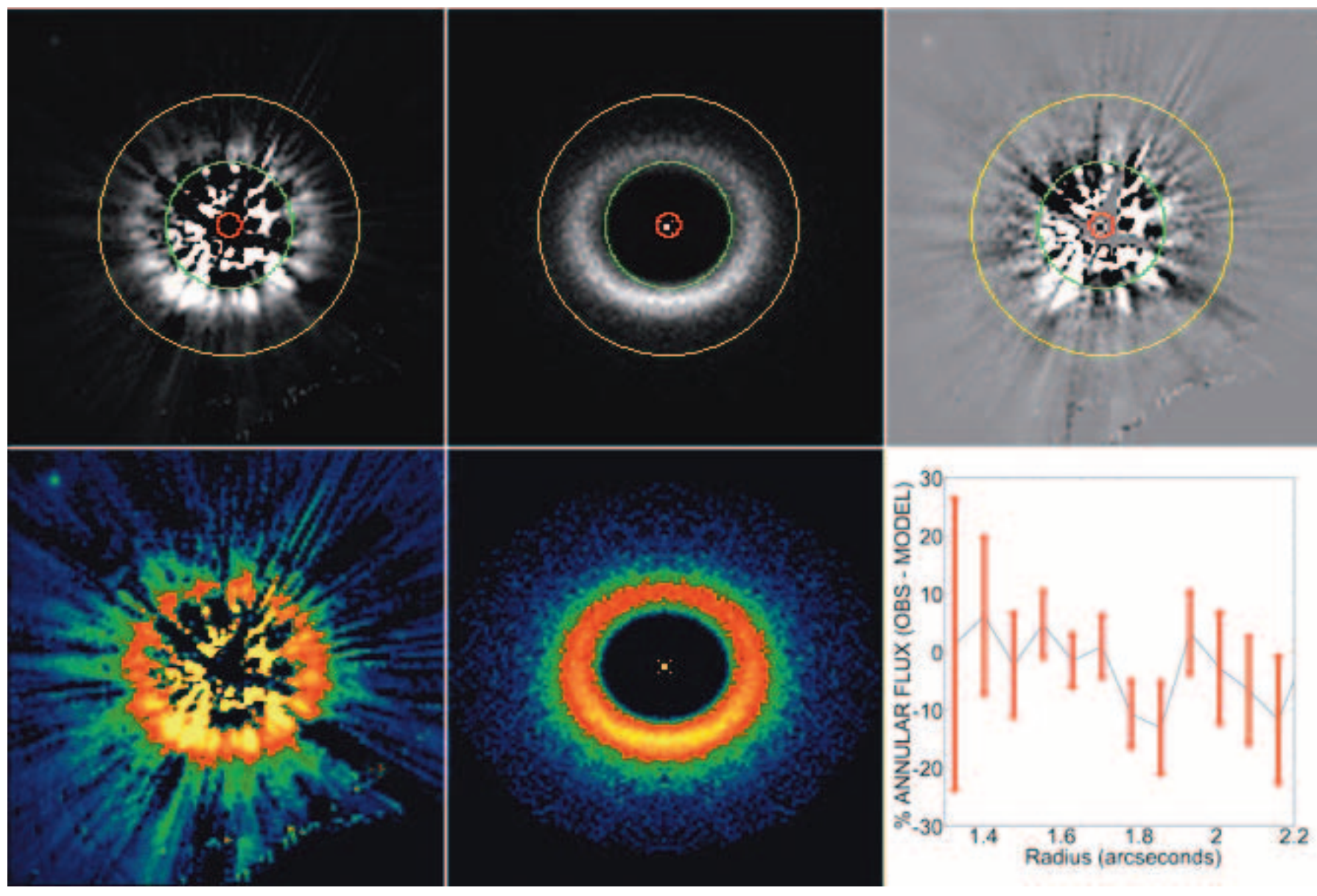

FIG. 13.- Representative $\chi^{2}$ (convergent minimization) fit of one of the MCFOST disk models (middle panels; the $\beta=1, \gamma=2, H_{0}=1$ AU case, as shown in Fig. 12) to the NICMOS observation of HD 181327 (left panels). The fitting region (in the $r \approx 1$ ".'3-2"3 annular zone) is bounded by the green and yellow circles overlaid in the top panels. Top, Linear displays $\left(0-1.5 \mathrm{mJy} \mathrm{arcsec}^{-2}\right.$; black to white) closely corresponding to the peak SB of the HD 181327 disk; bottom, same data displayed over the same dynamic range, but with a square root stretch (same color intensity scale, as shown in Fig. 12). Top right, Residuals remaining after subtracting the model image, shown in the linear $\left( \pm 1.5 \mathrm{mJy} \mathrm{arcsec}^{-2}\right)$ display; bottom right, residual flux densities and their $1 \sigma$ deviations about their respective medians in each 1 pixel (75.8 mas) wide annular zone, as a percentage of the predicted flux densities ( $\sim 10 \%$ over the region of the scattered-light ring).

minimum grain size, $a_{\min }$, and a maximum grain size, $a_{\max }$. We thus search for $\left(a_{\min }, \kappa\right)$ pairs consistent with the four constraints of color, $g_{\mathrm{HG}}$, IR excess, and blowout size limit (thus corresponding to the depletion timescale) identified above. In the following, the maximum grain size is assumed to be large enough that the results are not affected. The grain optical properties are calculated with the Mie theory valid for hard spheres and for astronomical silicates (optical constants from Weingartner \& Draine 2001).

The measured mean scattering asymmetry factor, $g_{\mathrm{HG}}=0.3 \pm$ 0.03 , in the $\mathrm{F} 110 \mathrm{~W}$ passband then reads

$$
g_{\mathrm{HG}}\left(a_{\min }, \kappa\right)=\int_{a_{\min }}^{a_{\max }} g(a) \pi a^{2} Q_{\mathrm{sca}} d n(a) / \sigma_{\mathrm{sca}}
$$

where $Q_{\text {sca }}$ is the dimensionless scattering efficiency, $\sigma_{\text {sca }}=$ $\int_{a_{\min }}^{a_{\max }} \pi a^{2} Q_{\text {sca }} d n(a)$, and $g(a)$ is the grain-size-dependent scattering asymmetry factor at $1.1 \mu \mathrm{m}$. As the disk is optically thin, the measured disk color depends on the ratio of scattering cross sections and phase functions at the two observed wavelengths. The mean scattering asymmetry factor at $0.6 \mu \mathrm{m}$, unfortunately, could not be measured from the ACS image. We therefore made the rough assumption that the phase function in the ACS passband is comparable to that found from the NICMOS $(1.1 \mu \mathrm{m})$ observations. In that case, the measured color corresponds directly to the mean scattering cross section ratio, $[\mathrm{F} 606 \mathrm{~W}]-[\mathrm{F} 110 \mathrm{~W}]=$ $2.5 \log \left(\sigma_{\text {sca }}[\mathrm{F} 606 \mathrm{~W}] / \sigma_{\text {sca }}[\mathrm{F} 110 \mathrm{~W}]\right)=+0.5 \pm 0.3$.

To further constrain the grain size, we used the surface density profile obtained in $\S 7$ to calculate infrared flux densities in the $\lambda>12 \mu \mathrm{m} I R A S^{21}$ and Spitzer MIPS bands (Table 2) as functions of $a_{\min }$ and $\kappa$ using the Augereau et al. (1999b) model. Fitting the SED leads to reduced $\chi^{2}$ contours in the $\left(a_{\min }, \kappa\right)$ parameter space, as illustrated in Figure 14. The best fit is obtained for minimum grain sizes close to $1 \mu \mathrm{m}$, in very good agreement with the radiation-pressure-induced minimum grain size. The SED fitting also reduces the range of possible $\kappa$ values, the best fit being obtained for a power-law index of $\approx-3.7$, quite close to the theoretical -3.5 power-law index, which holds for systems in collisional equilibrium (Dohnanyi 1969; Fig. 14, dashed line).

The ACS-NICMOS disk color is reproduced with only slightly smaller $\left(0.4 \mu \mathrm{m} \lesssim a_{\min } \lesssim 0.6 \mu \mathrm{m}\right)$ grains than those with sizes that best fit the thermal SED $\left(1 \mu \mathrm{m} \lesssim a_{\min } \lesssim 2 \mu \mathrm{m}\right)$. Nevertheless, these grains remain too large to reproduce the measured asymmetry factor $g_{\mathrm{HG}}$ (requiring $a_{\min } \lesssim 0.1 \mu \mathrm{m}$ grains in our formulation). In the frame of the Mie theory, the observed mean asymmetry factor can indeed be reproduced only when scattering

21 The $12 \mu \mathrm{m}$ emission detected by IRAS arises from the stellar photosphere, not the disk. 


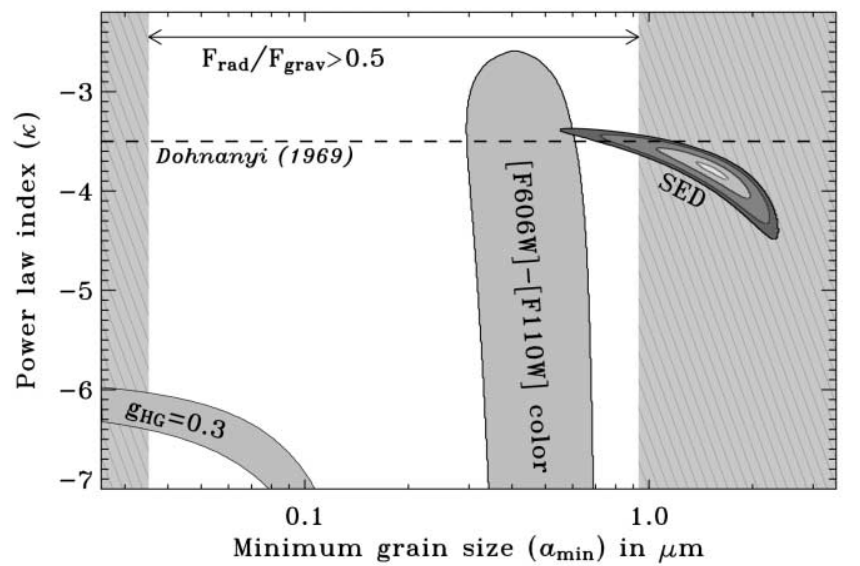

FIG. 14.- Minimum grain sizes and power-law indices suggested, separately, by the observed scattered-light disk asymmetry factor $(\S 5.3)$, the $[0.6]-[1.1] \mu \mathrm{m}$ color index of the grains $(\S 6.1)$, the thermal IR SED $(\S 4)$, and a theoretical radiation force blowout size limit ( $(8)$ presuming a single population of hard spherical silicate grains with $d n(a) \propto a^{\kappa} d a$. Optical constants from Weingartner \& Draine (2001) are adopted for Mie scattering by the grains. The radiation force and grain temperatures have been calculated with a NextGen atmosphere model spectrum $\left(T_{\text {eff }}=6450 \mathrm{~K}, \log g=4.5 ;\right.$ Hauschildt et al. 1999) scaled to match the observed $V$-band magnitude. SED fits corresponding to a reduced $\chi^{2}$ of $0.25,1,2.5$, and 3.5 (with corresponding probabilities of $78 \%, 37 \%, 8 \%$, and $3 \%$ ) are shown.

is dominated by very small grains, which is achieved when both $a_{\min }$ and $\kappa$ are extremely small. This inconsistency may relate to the fact that while we have calculated the asymmetry factor for hard silicate spheres (a simplifying assumption inappropriate for grains with more complex structures), the scattering phase function is very sensitive to the shape and structure of the grains. In addition, the HD 181327 disk may possess disk grains that (1) originate in more than a single population, (2) are radially segregated in distance from the star, and (3) have a grain size distribution not well represented by a single power law.

The grains that are predominantly responsible for HD 181327's thermal infrared excess may not be those that give rise to the circumstellar light scattered at 0.6 to $1.1 \mu \mathrm{m}$. In the case of the debris system circumscribing HR 4796A (Schneider et al. 1999), Augereau et al. (1999b) suggested a two-component dust population with large grains of ISM-like composition (moderate- porosity amorphous silicates) at the radius of the scattered-light disk and high-porosity crystalline grains close to the star. The thermal emission from HD 141569's circumstellar disk was shown by Marsh et al. (2002) to arise from within $1^{\prime \prime}(100 \mathrm{AU})$ of the star from 12 to $21 \mu \mathrm{m}$ imaging, while light scattered by the disk at 1.1 and $1.6 \mu \mathrm{m}$ (Weinberger et al. 1999; Augereau et al. 1999a) originates (and extends) significantly farther out. While HD 181327 's thermal SED is not inconsistent with the possibility of ISM-like grains (e.g., $\kappa \approx-3.5$, with $a_{\min }<1 \mu \mathrm{m}$; Mathis et al. 1977) in the scattered-light debris ring, the presumption of a single population of hard, spherical, silicate grains would be inapplicable for grains that have grown (evolved) from ISM-like structures and may be spatially (radially) segregated in distance from the star.

\section{DISCUSSION}

The inferred spatial distribution of dust detected about stars, from thermal infrared excesses (as measured from IRAS and Spitzer), often predicts central clearings or "holes" to explain the observed SEDs. Scattered-light imagery of circumstellar debris directly provides constraints on the spatial distribution of dust that are independent of the dust grain temperature in regions sufficiently far from the central star to be instrumentally probed with high spatial resolution. The central regions of dusty circumstellar disks that are nearly edge-on to the line of sight (e.g., $\beta$ Pictoris, AU Microscopii, and HD 32297) remain hidden at optical and near-IR wavelengths, due to the opacity of the dust. The inner regions of debris disk systems that are more inclined to the line of sight, such as HD 181327, are revealed directly and give support to the interpretation of inner holes from analyses of debris disk SEDs. While only a very small number of light-scattered images of circumstellar debris disks exist, the growing number allows intercomparison of the disk characteristics with stellar type and age (when sufficiently constrained; see Table 3; Fig. 15). With the notable exception of $\alpha$ PsA, which thus far has only been observed in scattered light with HST ACS, all of the debris disks listed in Table 3 have been observed with scattered-light imagery in the optical and the near-infrared. Very recently, two newly discovered, scattered-light disks around older (0.3-1 Gyr) stars, HD 53143 and HD 139664 (Kalas et al. 2006), have been reported, significantly adding to this still very limited sample.

TABLE 3

Circumstellar Debris Systems Imaged in Scattered Light

\begin{tabular}{|c|c|c|c|c|c|c|c|}
\hline Star & Spectral Type & $\begin{array}{l}\text { Age } \\
(\mathrm{Myr})\end{array}$ & $\begin{array}{c}r^{\mathrm{a}} \\
(\mathrm{AU})\end{array}$ & $f_{\mathrm{NIR}}(r>0 . " 3)$ & $L_{\mathrm{IR}} / L_{*}$ & Asymmetry & Image \\
\hline AU Mic ${ }^{\mathrm{b}}$ & M0 & $12-20$ & 210 & & 0.0004 & Wing tilt & Fig. $15 a$ \\
\hline HD $32297^{\mathrm{d}}$. & $\mathrm{A} 0$ & $\leq 10 ?$ & $>400$ & 0.0033 & 0.0027 & SB/W. tilt & Fig. $15 c$ \\
\hline HR 4796A & $\mathrm{A} 0$ & 8 & 70 & 0.0024 & 0.005 & Azimuthal & Fig. $15 d$ \\
\hline 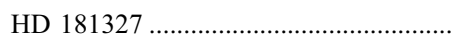 & $\mathrm{F} 5 / 6$ & 12 & 86 & 0.0017 & 0.0025 & No, $g \approx 0.3$ & Fig. $15 e$ \\
\hline HD 141569A f & HAeBe/B9.5 & 5 & 400 & 0.0025 & 0.0084 & Yes & Fig. $15 f$ \\
\hline
\end{tabular}

${ }^{\text {a }}$ The $r_{\mathrm{AU}}$ value reported is the scattered-light maximum extent detected for nearly edge-on disks (and HD 141569A), the radius of peak SB for ringlike disks (HR 4796A, HD 181327, and $\alpha$ PsA).

b AU Mic: Kalas et al. (2004).

c $\beta$ Pic: Kalas \& Jewitt (1995); $f_{\mathrm{NIR}}$ estimated from Kalas et al. (2000); $L_{\mathrm{IR}} / L_{*}$ from Decin et al. (2003).

${ }^{d}$ HD 32297: Schneider et al. (2005).

e HR 4796A: Schneider et al. (1999).

${ }^{\mathrm{f}}$ HD 141569A: Weinberger et al. (1999); for anisotropies, see Mouillet et al. (2001) and Clampin et al. (2003).

${ }^{g}$ HD 107146: Ardila et al. (2004); $g$ at $0.8 \mu \mathrm{m}$.

h $\alpha$ PsA: Kalas et al. (2005); $L_{\mathrm{IR}} / L_{*}$ from Decin et al. (2003). 


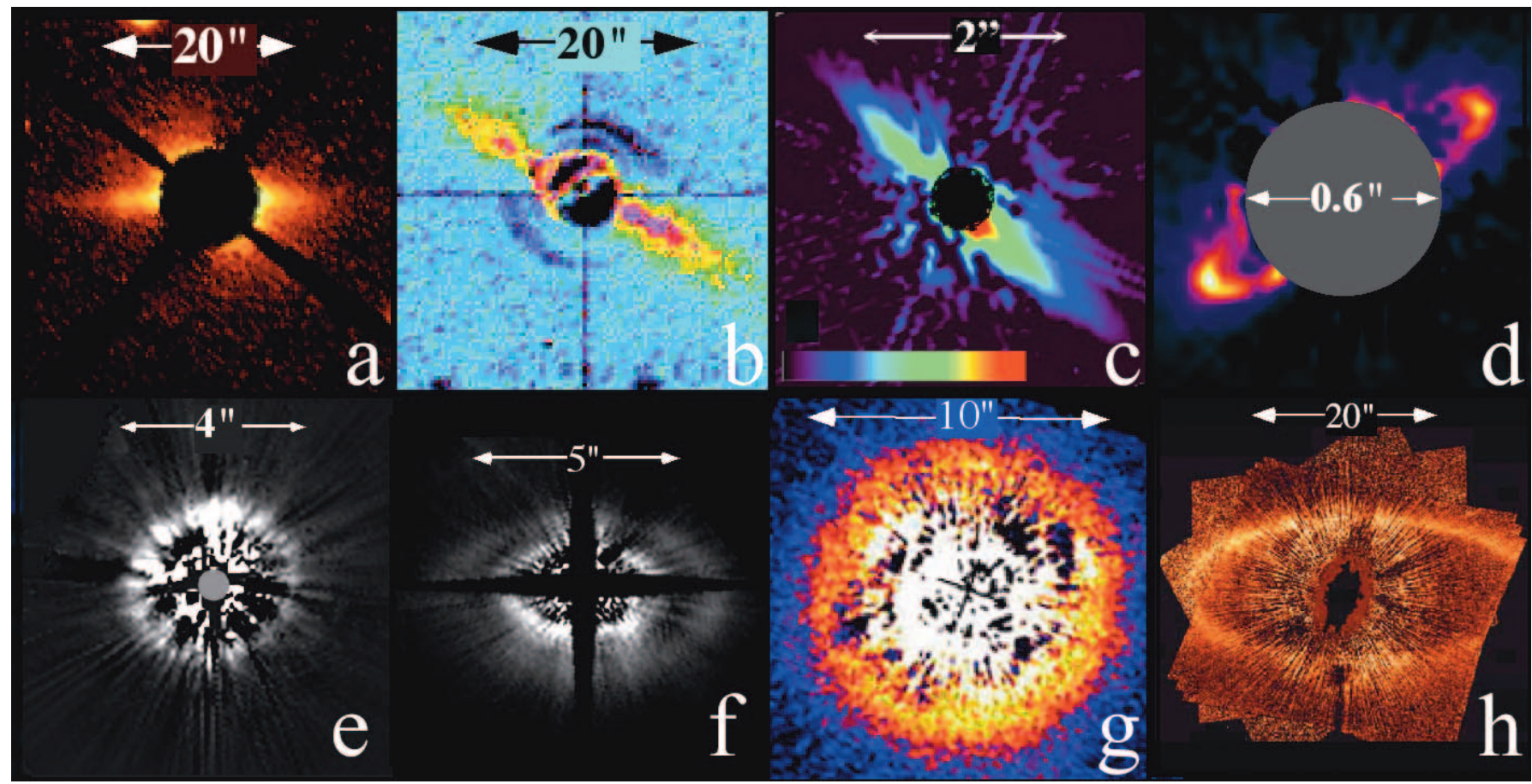

FIG. 15. - Circumstellar debris systems imaged in scattered light. (a) AU Mic; $(b) \beta$ Pic; $(c)$ HD 32297; $(d)$ HR 4796A; $(e)$ HD 181327; $(f)$ HD 141569; ( $g$ ) HD 107146; $(h) \alpha$ PsA. See Table 3 for details. Fig. $15 a$ reprinted with permission from Kalas et al., 2004, Science, 303 , 1990; C 2004 AAAS. Fig. 15b reprinted with permission from Smith \& Terrile, 1984, Science, 226, 1421; C) 1984 AAAS.

Of the debris disks imaged to date, the HR 4796A ringlike disk is, in many respects, the most morphologically similar to HD 181327 's. Both scattered-light disks appear as relatively narrow annuli, have similar near-IR scattering fractions and thermal IR excesses, and are of similar physical size (70 and $86 \mathrm{AU}$ in radii, respectively). Both stars, while not coeval (HR 4796A is a member of the TW Hya association, and HD 181327 is in the $\beta$ Pictoris moving group), are of similar ages ( $\sim 10 \mathrm{Myr})$ and spectral types.

Directionally preferential scattering, symmetric about the disk minor axis (i.e., with respect to the line of sight) is seen in the HD 181327 disk. Such nonisotropic scattering might be expected from circumstellar particles evolved from a primordial ISM-like population (comparable in size to, or larger than, the $1.1 \mu \mathrm{m}$ wavelength of the observation), and similar behavior is seen in the HR 4796A debris ring and in those of $\alpha$ PsA and HD 107146 as well.

These four ringlike debris disks vary by factors of $\sim 2$ in radius [from the already noted smaller HR 4796A and HD 181327 to the larger HD 107146 (133 AU; Ardila et al. 2004) and $\alpha$ PsA (145 AU; Kalas et al. 2005)] and $\sim 4$ in ring width-to-radius ratio ( $w: r$; from $\sim 0.17$, for both HR 4796A [Schneider et al. 2004] and $\alpha$ PsA, to 0.64 for the very broad HD 107146 ring, and, in between, HD 181327, at 0.38). The disks about HR 4796A and $\alpha$ PsA, both early A stars, are most disparate in radius, but most similar in $w: r$ ratios, suggesting a lack of simple scalability in ring geometries conflated (if not determined) instead by dynamical and kinematic processes in play. For example, in the case of HR 4796A, the outer radius of its disk may be truncated by the dynamical influence of its M-star companion (e.g., Artymowicz \& Lubow 1994). The sharp inner and more shallow outer edges of the illumination-corrected scattered-light radial profiles of HD 181327 (Fig. 7) and $\alpha$ PsA (Fig. 3 of Kalas et al. 2005) are remarkably similar, suggesting similar clearing and/or confinement mechanisms for the disk grains.
The photocenter of the $\alpha$ PsA dust ring is asymmetrically offset from the position of the central star. As suggested by Kalas et al. (2005), such an asymmetry may implicate the existence of one (or more) planetary perturbers. The HR 4796A debris ring exhibits an $\sim 20 \%$ SB asymmetry at the ring ansae at near-IR and optical wavelengths (Schneider \& Silverstone [2003a]; also seen in its mid-IR thermal emission, as reported by Telesco et al. 2000), with no photocentric offset in the position of the star relative to the ring ansae detectable in the scattered-light observations. Such a nonaxisymmetric SB asymmetry about the ring minor axis could also arise from a local enhancement (or depletion) in the dust density distribution due to perturbations from unseen, co-orbital, planetary-mass bodies (e.g., Ozernoy et al. 2000). However, alternative mechanisms, depending on the amount of residual gas in the system, could account for the ring structure itself (e.g., Klahr \& Lin 2005; Takeuchi \& Artymowicz 2001) without invoking planetary-mass companions.

The HD 181327 ring morphology is biaxially symmetric, centered on the star, and devoid of any readily apparent azimuthal SB anisotropies aside from those dependent on scattering phase angle. Such a ring structure could arise from gas drag in the absence of planets, although the gas-to-dust ratio in the HD 181327 disk is currently unknown.

\section{SUMMARY}

We have imaged a circumstellar debris disk about the $\sim 12 \mathrm{Myr}$ old F5/F6 V star and $\beta$ Pictoris moving group member HD 181327 in $1.1 \mu \mathrm{m}$ and $0.6 \mu \mathrm{m}$ light scattered by the disk grains. Our $1.1 \mu \mathrm{m}$ PSF-subtracted NICMOS coronagraphic observations reveal a bilaterally symmetric ringlike structure, $31^{\circ} .7 \pm 1{ }^{\circ} .6$ from face-on, with a major-axis P.A. of $107^{\circ} \pm 2^{\circ}$ east of north. We estimate the $1.1 \mu \mathrm{m}$ disk flux density in the region $1^{\prime \prime} .2<$ $r<5$ ". 0 to be $9.6 \pm 0.8 \mathrm{mJy}(0.17 \%$ of the total stellar light $)$, with $\sim 70 \%$ of the disk flux density contained within a $36 \mathrm{AU}$ 
wide annulus centered on the brightest zone at $r=86 \mathrm{AU}$ from the star, well beyond the blackbody-characterized 22 AU radius of the thermally emitting dust detected in the IRAS and Spitzer MIPS bands. The scattered-light disk appears to be centrally cleared (to within 1.2 of the star, the limiting region that can be effectively probed with our NICMOS observations) and centered on the star.

The SB of the ring of debris-scattered light is well represented by a Henyey \& Greenstein (1941) type scattering phase function with $g_{\mathrm{HG}}=0.3$. Modeling the disk, we find a vertical scale height $H_{0}=5 \%-10 \%$ of the $87.6 \mathrm{AU}$ radius of the maximum surface density at that radius. The observed dust ring explains the thermal IR excess emission above the stellar photospheric level measured by IRAS and Spitzer, provided that $a_{\min } \gtrsim 1 \mu \mathrm{m}$ with a grain size distribution $\propto a^{-3.7}$, presuming hard, spherical, silicate grains. With these grain parameters and the surface density profiles derived by direct inversion of the SB profiles obtained from the NICMOS imagery, we infer a total dust mass of $\approx 4 M_{\text {Moon }}$ $\left(\approx 0.05 M_{\oplus}\right)$ in the collision-dominated ring. Such grains, however, fail to reproduce the $g_{\mathrm{HG}}=0.3$ scattered-light asymmetry factor, suggesting a more complex (possibly mixed) population of disk grains. To better constrain the possible grain sizes and their radial distributions, in $H S T$ Cycle 15 we will obtain $2 \mu \mathrm{m}$ coronagraphic imaging polarimetry observations with NICMOS (a new, recently enabled, observing mode; Hines \& Schneider 2004) of the HD 181327 disk (GO program 10847).

Additional circumstellar light is seen from $2^{\prime \prime}$ to $4^{\prime \prime}(100$ $200 \mathrm{AU}$ ) in both $1.1 \mu \mathrm{m}$ NICMOS and $0.6 \mu \mathrm{m}$ ACS imagery, declining in $\mathrm{SB}$ as $\sim r^{-5}$ in both bands. The SB profile beyond $100 \mathrm{AU}$ is consistent with the blowout timescale for grains in the inner disk by radiation pressure, collisional grains with a size distribution as discussed, and low gas pressure in the disk. A lowSB diffuse outer halo $\left(V \approx 21.5 \mathrm{mag} \operatorname{arcsec}^{-2}\right)$ is seen in the ACS image extending as far as $\sim 9^{\prime \prime}(450 \mathrm{AU}$ ) to the north of the star.

We are grateful to F. J. Low and P. S. Smith of the Spitzer GTO (PID 72) team for providing MIPS imaging photometry of HD 181327. We thank Elizabeth Stobie (NICMOS Project, University of Arizona) for her significant contributions to this program through her development, implementation, enhancement, and support of analysis software used throughout the course of this study. We appreciate the commentary received from Eric Mamajek, particularly in regard to the age estimation for HD 181327 beyond its "guilt by association" as a presumed member of the $\beta$ Pictoris moving group, as reflected in our discussion in $\S \S 1$ and 6.3 of this paper. This investigation was based on observations made with the NASA/ESA Hubble Space Telescope, obtained at the Space Telescope Science Institute (STScI), which is operated by the Association of Universities for Research in Astronomy, Inc., under NASA contract NAS 5-26555. These observations are associated with programs GO 10177 and GTO/9987. Support for program GO 10177 was provided by NASA through a grant from STScI. S. Wolf was supported by the German Research Foundation (DFG) through the Emmy Noether grant WO 857/2-1. J.-C. Augereau, C. Pinte, and F. Ménard acknowledge financial support from the Programme National de Physique Stellaire (PNPS) of CNRS/INSU, France. We acknowledge the use of the SIMBAD database.
Ardila, D. R., et al. 2004, ApJ, 617, L147

Artymowicz, P., \& Lubow, S. H. 1994, ApJ, 421, 651

Augereau, J. C., \& Beust, H. 2006, A\&A, 455, 987

Augereau, J. C., Lagrange, A. M., Mouillet, D., \& Menard, F. 1999a, A\&A, 350, L51

Augereau, J. C., Lagrange, A. M., Mouillet, D., Papaloizou, J. C. B., \& Grorod, P. A. 1999 b, A\&A, 348, 557

Augereau, J. C., Nelson, R. P., Lagrange, A. M., Papaloizou, J. C. B., \& Mouillet, D. 2001, A\&A, 370, 447

Aumann, H. H., et al. 1984, ApJ, 278, L23

Backman, D. E., \& Paresce, F. 1993, in Protostars and Planets III, ed. E. H. Levy \& J. I. Lunine (Tucson: Univ. Arizona Press), 1253

Baraffe, I., Chabrier, G., Allard, F., \& Hauschildt, P. 1998, A\&A, 337, 403

Bohlin, R., Hartig, G., \& Sparks, W. 2003, STScI Instument Science Report ACS 02-03 (Baltimore: STScI), http://www.stsci.edu/hst/acs/documents/isrs/ isr0203.pdf

Buitrago, J., \& Mediavilla, E. 1986, A\&A, 162, 95

Burgasser, A. J., Reid, I. N., Siegler, S., Close, L., Allen, P., Lowrance, P., \& Gizis, J. 2006, in Protostars and Planets V, ed. B. Reipurth, D. Jewitt, \& K. Keil (Tucson: Univ. Arizona Press), in press

Chauvin, G., et al. 2003, A\&A, 404, 157

Chen, C., et al. 2006, ApJS, 166, 351

Clampin, M., et al. 2003, AJ, 126, 385

Close, L. M., Siegler, N., Freed, M., \& Biller, B. 2003, ApJ, 587, 407

Cutri, R. M., et al. 2003, VizieR Online Data Catalog, 2246, 0

D’Antona, F., \& Mazzitelli, I. 1997, Mem. Soc. Astron. Italiana, 68, 807

Decin, G., Dominik, C., Waters, L. B. F. M., \& Waelkens, C. 2003, ApJ, 598, 636

Dohnanyi, J. W. 1969, J. Geophys. Res., 74, 2531

Gilliland, R. 2004, STScI Instrument Science Report ACS 04-01 (Baltimore: STScI), http://www.stsci.edu/hst/acs/documents/isrs/isr0401.pdf

Gonzaga, S., et al. 2005, ACS Instrument Handbook, Version 6.0 (Baltimore: STScI)

Grady, C. A., et al. 2001, AJ, 122, 3396

Hauschildt, P. H., Allard, F., \& Baron, E. 1999, ApJ, 512, 377

Henyey, L. G., \& Greenstein, J. L. 1941, ApJ, 93, 70

Hines, D. C., \& Schneider, G. 2004, BAAS, 205.0504

Kalas, P., Graham, J. R., Beckwith, S. V. W., Jewitt, D. C., \& Lloyd, J. P. 2002, ApJ, 567, 999

\section{REFERENCES}

Kalas, P., Graham, J. R., \& Clampin, M. 2005, Nature, 435, 1067

Kalas, P., Graham, J. R., Clampin, M. C., \& Fitzgerald, M. P. 2006, ApJ, 637, L57

Kalas, P., \& Jewitt, D. 1995, AJ, 110, 794

Kalas, P., Larwood, J., Smith, B. A., \& Schultz, A. 2000, ApJ, 530, L133

Kalas, P., Liu, M. C., \& Matthews, B. C. 2004, Science, 303, 1990

Klahr, H., \& Lin, D. N. C. 2005, ApJ, 632, 1113

Krist, J., \& Hook, R. N. 1997, in The 1997 HST Calibration Workshop with a New Generation of Instruments, ed. S. Casertano, R. Jedrzejewski, \& T. Keyes (Baltimore: STScI), 192

Krist, J., et al. 2005, AJ, 129, 1008

Lecavelier des Étangs, A., Vidal-Madjar, A., \& Ferlet, R. 1996, A\&A, 307, 542

Lowrance, P. J., et al. 2000, ApJ, 541, 390

2005, AJ, 130, 1845

Mamajek, E., et al. 2004, ApJ, 612, 496

Mannings, V., \& Barlow, M. J. 1998, ApJ, 497, 330

Marsh, K. A., Silverstone, M. D., Becklin, E. E., Koerner, D. W., Werner, M. W., Weinberger, A. J., \& Ressler, M. E. 2002, ApJ, 573, 425

Mathis, J., Rumpl, W., \& Nordsieck, K. 1977, ApJ, 217, 425

Metchev, S. A., et al. 2005, ApJ, 622, 451

Mouillet, D., Lagrange, A. M., Augereau, J. C., \& Ménard, F. 2001, A\&A, 372, L61

Neuhäuser, et al. 2003, Astron. Nachr., 324, 535

Noll, K., et al. 2004, NICMOS Instrument Handbook, Version 7.0 (Baltimore: STScI)

Nordstrom, B., et al. 2004, A\&A, 418, 989

Ozernoy, L. M., Gorkavyi, N. N., Mather, J. C., \& Taidakova, T. A. 2000, ApJ, 537, L147

Palla, F., \& Stahler, S. W. 2001, ApJ, 553, 299

Pantin, E., \& Lagage, P.-O. 1996, in The Role of Dust in the Formation of Stars, ed. H. U. Käufl \& R. Siebenmorgen (Berlin: Springer)

Perryman, M. A. C., et al. 1997, A\&A, 323, L49

Pinte, C., et al. 2006, A\&A, in press (astro-ph/0606550)

Schneider, G., Becklin, E. E., Smith, B. A., Weinberger, A. J., Silverstone, M., \& Hines, D. C. 2001, AJ, 121,525

Schneider, G., Cotera, A. S., Silverstone, M. D., \& Weinberger, A. J. 2004, in ASP Conf. Ser. 324, Debris Disks and the Formation of Planets (San Francisco: ASP), 247 
Schneider, G., \& Silverstone, M. 2003a, in ASP Conf. Ser. 291, Hubble's Science Legacy: Future Optical/Ultraviolet Astronomy from Space, ed. K. R. Sembach, et al. (San Francisco: ASP), 69

Schneider, G., Silverstone, M., \& Hines, D. C. 2003, BAAS, 203, 4602S

Schneider, G., \& Silverstone, M. D. 2003b, Proc. SPIE, 4860, 1 2005, ApJ, 629, L117 (SSH05)

Schneider, G., et al. 1999, ApJ, 513, L127

Smith, B. A., \& Terrile, R. J. 1984, Science, 226, 1421
Takeuchi, T., \& Artymowicz, P. 2001, ApJ, 557, 990

Telesco, C., et al. 2000, ApJ, 530, 329

Thébault, P., \& Augereau, J.-C. 2005, A\&A, 437, 141

Weinberger, A., et al. 1999, ApJ, 525, L53

Weingartner, J. C., \& Draine, B. T. 2001, ApJ, 548, 296

Zuckerman, B., \& Song, I. 2004, ApJ, 603, 738

Zuckerman, B., Song, I., Bessell, M. S., \& Webb, R. A. 2001, ApJ, 562, L87

Zuckerman, B., \& Webb, R. A. 2000, ApJ, 535, 959 